AEI-2003-108

UCLA/03/TEP/41

ITFA-2004-09

hep-th/0403035

\title{
The Off-shell M5-brane and Non-Perturbative Gauge Theory
}

\author{
Jan de Boer ${ }^{\star}$ and Sebastian de Haro ${ }^{\dagger}$ \\ ${ }^{\star}$ Institute for Theoretical Physics \\ University of Amsterdam \\ Valckenierstraat 65, 1018 XE Amsterdam, The Netherlands \\ jdeboer@science.uva.nl \\ † Max-Planck-Insitut für Gravitationsphysik \\ Albert-Einstein-Institut \\ 14476 Golm, Germany \\ sdh@aei.mpg.de
}

\begin{abstract}
M5-branes wrapping a holomorphic curve in a Calabi-Yau manifold can be used to construct four-dimensional $\mathcal{N}=1$ gauge theories. In this paper we will consider M5-brane configurations corresponding to $\mathcal{N}=2$ theories broken to $\mathcal{N}=1$ by a superpotential for the adjoint scalar field. These M5-brane configurations can be obtained by lifting suitable intersecting brane configurations in type IIA, or equivalently by T-dualizing IIB configurations with branes and/or fluxes. We will show that turning on non-trivial expectation values for the glueball superfields corresponds to non-holomorphic deformations of the M5-brane. We compute the superpotential and show it agrees with that computed by Dijkgraaf and Vafa. Several aspects of the gauge theory, such as the appearance of non-holomorphic one-forms with integer periods on the SeibergWitten curve, have a natural interpretation from the M5-brane point of view. We also explain the interpretation of the superpotential in terms of the twisted $(2,0)$ theory living on the fivebrane.
\end{abstract}




\section{Introduction}

Recently, major progress has been achieved in understanding the interconnections between Fterms in $4 \mathrm{~d} \mathcal{N}=1 \mathrm{SYM}$ theory, topological string theory, matrix models, and integrable systems, starting with [1]. In particular, a remarkably simple prescription to determine the nonperturbative superpotential of certain $\mathcal{N}=1$ gauge theories in terms of a matrix integral was given in [1]. The superpotential is expressed as a function of gluino condensate superfields $S_{i}$, and it is intriguing that one is in some sense doing off-shell computations, i.e. away from the supersymmetric minima of the superpotential.

One aspect of this off-shell construction that has been less well understood is the need to include non-holomorphic quantities when one goes off-shell. In the type IIB construction of these gauge theories [2] one employs a Calabi-Yau manifold with three-form flux, and the superpotential is of the form $W=\int H \wedge \Omega$, with $H=H_{R}+\tau H_{N S}$ the three-form flux and $\Omega$ the holomorphic three-form. Superficially, the entire superpotential comes from the antiholomorphic part of the flux. This parallels the discussion in field theory [3, 4], where it was shown that the off-shell variation of the superpotential involves a closed one-form $T$ that is not holomorphic. This is because once one has fixed the A- and B-periods of a holomorphic differential, one cannot independently turn on expectation values for the gaugino condensates as this corresponds to varying the moduli of the underlying Riemann surface. If one wants to do the latter, one needs to consider a non-holomorphic one-form $T$.

In the present paper we wish to study the non-perturbative physics and in particular the superpotential of deformed $\mathcal{N}=2$ gauge theories from the M-theory perspective. The description of $\mathcal{N}=2$ theories in terms of an M5-brane wrapped on the Seiberg-Witten curve was first found in [5], by lifting a suitable intersecting brane configuration in type IIA to M-theory. This was generalized to mass-deformed $\mathcal{N}=2$ and pure $\mathcal{N}=1$ theories in $[6,7]$. For a review and applications of intersecting brane configurations to gauge dynamics see e.g. [8]. The M5-brane configuration for $\mathcal{N}=2$ theories deformed by a general superpotential for the adjoint scalar field was found in [9], again by lifting a suitable brane configuration from type IIA to M-theory. The process of lifting a brane configuration from type IIA to M-theory is T-dual to the large $N$ transition of [10]: the type IIA configuration with intersecting branes is dual to a resolved type IIB geometry with D5 branes wrapping $\mathbb{P}^{1}$ 's, whereas the M-theory configuration is dual to the deformed type IIB geometry with fluxes (see e.g. [11]). The field theory living on the worldvolume of the M5-brane is not quite that of an ordinary supersymmetric four-dimensional gauge theory, due to the existence of additional light degrees of freedom coming from the KK-modes of the M-theory circle. For this reason the field theory is sometimes called MQCD instead of QCD. However, as long as we consider BPS-like quantities such as the F-terms in the low-energy effective field theory the M5-brane yields the same answer as the gauge theory. The agreement breaks down for non-BPS quantities such as the Kähler potential of $\mathcal{N}=1$ theories and higher derivative terms in the $\mathcal{N}=2$ theory [12].

Since the superpotential is a holomorphic quantity one should be able to compute it directly from the M5-brane configuration. In [5] an expression for the effective superpotential was proposed and evaluated in a few examples. This expression is rather cumbersome to work with (some more examples are worked out in [13]). Here we will compute this superpotential in more general cases, and hopefully shed some light on its interpretation. In particular, the M-theory 
setup gives a purely geometric interpretation of all the quantities involved in the Dijkgraaf-Vafa proposal, and can be seen as a pure geometric dual of the type IIB setup with fluxes: all information is encoded in a surface embedded in $\mathbb{R}^{5} \times S^{1}$. We will give a precise off-shell definition of the superpotential and we will see that it reproduces the Dijkgraaf-Vafa result for 4d SYM theory. As it will turn out, going off-shell is quite subtle, as the embedding of the fivebrane in the Calabi-Yau will no longer be holomorphic. We will also discuss how the superpotential arises from the kinetic term for the scalars in the $(2,0)$ theory living on the world-volume of the fivebrane via deconstruction. This generalizes the relation between the $(2,0)$ theory and $\mathcal{N}=2$ SYM $[14,12]$ to the case of $\mathcal{N}=1$, and in particular to the precise computation of the F-terms.

In the $\mathcal{N}=2$ case the fivebrane worldvolume is of the form $\mathbb{R}^{4} \times \Sigma$, where the Riemann surface $\Sigma$ is holomorphically embedded in $\mathbb{R}^{3} \times S^{1}$. Thus there is a non-trivial background value for one of the complex transverse scalars. In the case of $\mathcal{N}=1$, two transverse scalars, which we call $t$ and $w$, have a non-trivial configuration, and so we will take $\Sigma$ to be embedded in $\mathbb{R}^{5} \times S^{1}$ with complex coordinates $t, w, v$. The complex coordinate $t$ takes values in $\mathbb{C}^{*}$ and parametrizes the cylinder $\mathbb{R} \times S^{1}$, whereas $v, w$ take values in the complex plane. On-shell, the embedding is given by two algebraic equations [9]:

$$
\begin{aligned}
t^{2}-2 P_{N}(v) t+\Lambda_{N=2}^{2 N} & =0 \\
w^{2}-2 W_{m}^{\prime}(v) w-\tilde{f}_{m-1}(v) & =0 .
\end{aligned}
$$

where $P_{N}, W_{m}^{\prime}$ and $\tilde{f}_{m-1}$ are polynomials of degrees $N, m, m-1$. In order for $t$ and $w$ to be meromorphic with a prescribed singularity structure, the following factorization formulas must also hold:

$$
\begin{aligned}
P_{N}(v)^{2}-\Lambda_{N=2}^{2 N} & =S_{N-n}^{2}(v) y(v) \\
W_{m}^{\prime}(v)^{2}+\tilde{f}_{m-1}(v) & =H_{m-n}^{2}(v) y(v)
\end{aligned}
$$

for suitable polynomials $S, H$, and $y(v)$ describes a Riemann surface of genus $n-1$ :

$$
y^{2}=G_{n}^{2}(v)+f_{n-1}(v) .
$$

By counting parameters we see that (2) has the same number of free parameters as there are equations for fixed $W_{m}^{\prime}$. Thus given a superpotential $W$, these equations will generically have a discrete set of solutions for all other coefficients. This factorization agrees with the results from field theory $[9,2]$. We propose the following relation of the embedding coordinates $t$ and $w$ to the gauge theory quantities:

$$
\begin{array}{rlrl}
N_{i} & =\frac{1}{2 \pi i} \oint_{A_{i}} \frac{\mathrm{d} t}{t} & \tau+\tau_{i}=\frac{1}{2 \pi i} \int_{\hat{B}_{i}} \frac{\mathrm{d} t}{t} \\
S_{i}=\frac{1}{2 \pi i} \oint_{A_{i}} w \mathrm{~d} v & \frac{\partial \mathcal{F}}{\partial S_{i}}=\oint_{B_{i}} w \mathrm{~d} v .
\end{array}
$$

$N_{i}$ is the classical $U(N) \rightarrow \prod_{i=1}^{n} U\left(N_{i}\right)$ symmetry breaking pattern, $\tau$ is the $U(1) \subset U(N)$ coupling constant [2] (which is the bare coupling constant of the YM theory), the $\tau_{i}$ are integers that describe generalized theta-angles [3], $S_{i}$ are the corresponding gluino condensates, and $\frac{\partial \mathcal{F}}{\partial S_{i}}$ are the dual magnetic variables. $\hat{B}_{i}$ are the non-compact $B$-periods, i.e. $\hat{B}_{i}=B_{i}+B_{n}$ where 
$B_{i}$, the compact periods, and the non-compact period $B_{n}$, are defined in appendix A. These relations become obvious once we realize that $\mathrm{d} t / t$ and $w \mathrm{~d} v$ are related to the gauge theory and matrix model resolvents. Namely

$$
\frac{\mathrm{d} t}{t}=\operatorname{Tr}_{\text {gauge theory }}\left(\frac{\mathrm{d} v}{v-\Phi}\right)
$$

is the gauge theory resolvent $[15,3]$, whereas

$$
w \mathrm{~d} v=2 \operatorname{Tr}_{\text {matrix theory }}\left(\frac{\mathrm{d} v}{v-M}\right)=-\frac{1}{16 \pi^{2}} \operatorname{Tr}_{\text {gauge theory }}\left(\frac{W_{\alpha} W^{\alpha} \mathrm{d} v}{v-\Phi}\right)
$$

$[1,15]$. Thus the M5-brane treats the gauge theory and matrix model on a symmetric footing: the gauge theory curve is the projection in the $t, v$-plane, whereas the matrix model curve is the projection in the $t, w$ plane. The above relations are valid on-shell. To go off-shell, we want to be able to vary the $S_{i}$ 's at will, keeping the periods of $\mathrm{d} t / t$ fixed. From the M5-brane point of view, this corresponds to deforming the way the Riemann surface is embedded in $\mathbb{R}^{5} \times S^{1}$. Thus we look for a continuous family of deformations of $\Sigma$. However, we will find that such deformations do not exist unless they are non-holomorphic, which makes $t=t(v, \bar{v})$ a function of both $v$ and $\bar{v}$. This precisely parallels the discussion in the gauge theory [4], where it was found that the one-form $T$, which corresponds to our $\mathrm{d} t / t$, had to be non-holomorphic. We will analyze in detail this non-holomorphic embedding, and we will find that it can be solved only if we include also a log-normalizable anti-holomorphic deformation.

Notice that only $t$, and not $w$, will be non-holomorphic off-shell. Indeed, the fact that $t$ parametrizes a cylinder, $t=\exp \left(-\left(x^{6}+i x^{10}\right) / R\right)$ with $x^{10}$ the compact M-theory direction with radius $R$, implies that the periods of $\mathrm{d} t / t$ have to be integers, and this gives an interesting new purely geometrical interpretation of the integrality of the periods of $T$ around the compact cycles. Once we go off-shell we only change the periods of $w \mathrm{~d} v$ while keeping its asymptotic behavior fixed. Under T-duality to the IIB description, the one-form $\mathrm{d} t / t$ maps to the flux three-form $H$, while the one-form $w \mathrm{~d} v$ maps to the complex structure three-form $\Omega$. In this way the deformations of the Riemann-surface are in one-to-one correspondence with complex structure deformations of the T-dual Calabi-Yau.

The main goal in this paper is to study the non-holomorphic deformations of the M5-brane and to rederive the gauge theory superpotential from this perspective. In particular we will encounter several different definitions of the superpotential that all turn out to be equivalent. The outline of this paper is as follows. In section 2 we will briefly review the brane construction of the gauge theory and its lift to M-theory. We will also describe the way in which the worldvolume is twisted and see that this may provide a natural framework to understand a peculiar auxiliary supermultiplet found in [15]. In section 3 we discuss the non-holomorphic embedding of the M5-brane, and use this to compute the expression for the superpotential proposed in [7]. In section 4 we consider the non-holomorphic geometries in some more detail, and in section 5 we discuss some further aspects of the M5-brane world-volume theory, such as the "deconstruction" interpretation of the superpotential. Several open problems are given at the end in section 6 . 


\section{The on-shell M5-brane construction}

It is well-known that an M5-brane with worldvolume $\mathbb{R}^{4} \times \Sigma$ preserves half the supersymmetry if $\Sigma$ is holomorphically embedded in a 2-dimensional complex space, for example in $\mathbb{R}^{3} \times S^{1}$. The fluctuations of this Riemann surface in $\mathbb{R}^{3} \times S^{1}$ are then described by a single complex scalar superfield. In order to break supersymmetry to $\mathcal{N}=1$ we need to consider a configuration where two complex scalars are active, which happens for instance when $\Sigma$ is a holomorphic two-cycle in a Calabi-Yau $X$. In fact, the requirements of meromorphy of the embedding and a choice of boundary conditions at infinity are enough to determine the fivebrane geometry. In this section we will review this on-shell description.

\subsection{The type IIA picture}

It is useful to start from type IIA and then lift to M-theory. The $\mathcal{N}=2 S U(N)$ super-Yang Mills theory can be engineered with $N$ D4-branes suspended between two NS5-branes [5]. Our notation will be as follows. We denote the coordinates along the worldvolume of the D4-branes by $x^{0}, x^{1}, x^{2}, x^{3}, x^{6}$, and those of the NS 5 -branes by $x^{0}, x^{1}, x^{2}, x^{3}, x^{4}, x^{5}$. The NS 5 -branes are at a finite distance from each other along the $x^{6}$-direction. Classically, the positions of the D4branes in transverse space are fixed, but quantum mechanically they are allowed to fluctuate, becoming scalar fields on the brane with fixed boundary conditions at the ends, where the NS 5-branes sit. Together with $x^{6}$ and the gauge field component along $x^{6}$, the scalar fields are parametrized by $v=x^{4}+i x^{5}, w=x^{7}+i x^{8}$. The classical rotation invariances along the $x^{4}, x^{5}$ and $x^{7}, x^{8}, x^{9}$ directions, $U(1)_{4,5}$ and $S U(2)_{7,8,9}$, respectively, correspond to the classical $U(1)$ and $S U(2) \mathrm{R}$-symmetries of the 4-dimensional theory on the D4-branes.

To obtain a configuration with $\mathcal{N}=1$ supersymmetry with arbitrary superpotential for the adjoint scalar field, we consider the brane construction in [9], in which the first NS 5brane extends along $x^{0}, x^{1}, x^{2}, x^{3}, x^{4}, x^{5}$, whereas $N-1$ NS' 5 -branes have worldvolume coordinates $x^{0}, x^{1}, x^{2}, x^{3}, x^{7}, x^{8}$. The D4-branes between them have worldvolume coordinates $x^{0}, x^{1}, x^{2}, x^{3}, x^{6}$. (Constructions involving non-generic superpotentials were also given in [16, $17,6,7])$. As shown in [9], adding a superpotential of the form

$$
W_{\text {tree }}=\sum_{k=2}^{N} \mu_{k} \operatorname{Tr} \Phi^{k}
$$

corresponds to bending the NS' 5-branes, allowing them to stretch in the $(v, w)$ plane. Classically, the minima of (7) give the separation of the NS' 5 -branes in the $v$-direction. It will however be more convenient to view the NS' 5 -branes as a single NS 5 -brane bent in the $w$-direction, rather than $N-1$ NS' 5 -branes bent in the $v$-direction, since this is the appropriate configuration for finite values of the $\mu_{k}[9]$.

\section{$2.2 \quad$ Lifting to M-theory}

The M-theory configuration [5] consists of a single M5-brane wrapped on a 2-cycle $\Sigma$ of a CalabiYau $X=\mathbb{R}^{5} \times S^{1}$. $\Sigma$ will generically be a Riemann surface of genus $N-1$. The world-volume of the M5-brane is thus $\mathbb{R}^{4} \times \Sigma$, and the total space is $\mathbb{R}^{4} \times X \times \mathbb{R}$; as a separate problem one 
can also take the total space to be $\mathbb{R}^{4} \times X \times S^{1}$, so that one can T-dualize from IIA to the IIB picture. This is however not quite the standard $4 \mathrm{~d}$ gauge theory, since it corresponds to having a compact $x^{9}$-direction. The field theory lives on the $\mathbb{R}^{4}$ spanned by $x^{0}, \ldots, x^{3}$, whereas $t, v, w$ are complex coordinates $v=x^{4}+i x^{5}, w=x^{7}+i x^{8}, t=e^{s}=e^{-R^{-1}\left(x^{6}+i x^{10}\right)}$ on $X$. They can be used to describe the transverse fluctuations of $\Sigma$ embedded in $X$.

As shown in [5], for $\mathcal{N}=2$ theories, $\Sigma$ is a Riemann surface given by an algebraic equation in the $(v, t)$ plane, the $\mathcal{N}=2$ Seiberg-Witten curve:

$$
t^{2}-2 P_{N}(v) t+\Lambda_{N=2}^{2 N}=0
$$

where $P_{N}(v)$ is a polynomial of order $N$ in $v \cdot t$ is related to the more commonly used variable $y$, defined by

$$
y^{2}=P_{N}^{2}(v)-\Lambda_{N=2}^{2 N},
$$

by $t=y+P_{N}(v)$. However, in the remainder of the paper, we will use $y(v)$ not to denote the variable defined by the above relation, but by the relation

$$
S_{N-n}^{2}(v) y^{2}=P_{N}^{2}(v)-\Lambda_{N=2}^{2 N},
$$

where $S_{N-n}(v)$ contains all the double zeroes of $P_{N}^{2}(v)-\Lambda_{N=2}^{2 N}$.

The M-theory configuration corresponding to the IIA set-up with a superpotential $W_{\text {tree }}$ was constructed in [9]. The left NS 5-brane corresponds to the asymptotic region $v \rightarrow \infty, t \sim v^{N}$, and the right NS 5-brane to $v \rightarrow \infty, t \sim \Lambda_{N=2}^{2 N} v^{-N}$. One imposes the following boundary conditions on $w$ at infinity:

$$
\begin{array}{ll}
w \rightarrow 2 W_{m}^{\prime}(v) & \text { as } \quad v \rightarrow \infty, \quad t \sim \Lambda_{N=2}^{2 N} v^{-N} \\
w \rightarrow 0 & \text { as } \quad v \rightarrow \infty, \quad t \sim v^{N},
\end{array}
$$

where $W_{m}^{\prime}(v)$ is a polynomial of order $m$ that corresponds to the field theory tree level superpotential. Notice that here $m$ does not have to be equal to $N-1$. Physically, the above means that we bend one of the two NS 5-branes in such a way that the masses of open strings stretched between a straight D4 brane and one of the two 5-branes is proportional to $W_{m}^{\prime}(v)$, in agreement with the classical properties of the field theory deformed by the superpotential (7). As we will see, these two regions correspond to the two "branches", "quantum" and "classical", respectively, in [4].

There is an alternative choice of boundary conditions, but this only corresponds to interchanging the two NS 5-branes. More generally, we could impose $w \rightarrow a W_{m}^{\prime}(v)$ on one branch, and $w \rightarrow b W_{m}^{\prime}(v)$ on another, with $a-b=2$, but that will not affect any of the F-term physics. It might have some effect on non-holomorphic terms in the $4 \mathrm{~d}$ effective action but we will not explore that here.

In the analysis of [9], the fact that the form of the $\mathcal{N}=2$ curve remains unchanged under the breaking to $\mathcal{N}=1$ means that the embedding coordinate $t$ still satisfies equation (8). Breaking to $\mathcal{N}=1$, however, does require this curve to factorize, as we will now see. As for $w$, which was zero in the $\mathcal{N}=2$ case - its form can be fixed from the requirement that it is a rational function of $t$ and $v$ satisfying the above boundary conditions. The analysis then leads to the following 
conclusions (for details we refer to [9]). The requirement that $w$ be a rational function of $t, v$ with no other poles than at infinity, implies that it can be written in the following form:

$$
w(t, v)=N_{m}(v)+\frac{H_{m-n}(v)}{S_{N-n}(v)}\left(t-P_{N}(v)\right),
$$

where $N_{m}(v)$ and $H_{m-n}(v)$ are as yet arbitrary polynomials of the order indicated, $P_{N}(v)$ is the polynomial appearing in the $\mathcal{N}=2$ curve, and $S_{N-n}(v)$ is the set of double roots in the factorization formula of the Seiberg-Witten curve:

$$
P_{N}^{2}(v)-\Lambda_{N=2}^{2 N}=S_{N-n}^{2}(v) Q_{2 n}(v)
$$

so that $S_{N-n}$ and $Q_{2 n}$ are given by

$$
\begin{aligned}
S_{N-n}(v) & =\prod_{i=1}^{N-n}\left(v-s_{i}\right) \\
Q_{2 n}(v) & =\prod_{i=1}^{2 n}\left(v-q_{i}\right)
\end{aligned}
$$

where all $q_{i}$ 's are different. Using the expression for $t$ in (8), $w$ can also be rewritten as

$$
w\left(t_{ \pm}(v), v\right)=N_{m}(v) \pm H_{m-n}(v) \sqrt{Q_{2 n}(v)}
$$

depending on whether we choose the plus or the minus solution. These two different choices correspond to the two different asymptotic regions.

One now has to impose the boundary conditions on $w$. The first boundary condition, $t=$ $t_{-}(v) \sim v^{N}$ as $v \rightarrow \infty$, completely fixes $N_{m}(v)$ :

$$
N_{m}(v)=\left[H_{m-n}(v) \sqrt{Q_{2 n}(v)}\right]_{+},
$$

where $[Q(v)]_{+}$is the part of $Q$ with non-negative powers of $v$, in a power series expansion around $v=\infty$. Substituting this in $w$, we get in the second asymptotic region

$$
w\left(t_{+}(v), v\right)=2\left[H_{m-n}(v) \sqrt{Q_{2 n}(v)}\right]_{+}+\mathcal{O}\left(\frac{1}{v}\right)
$$

as $v \rightarrow \infty, t=t_{+}(v) \sim v^{-N}$. The second boundary condition thus amounts to

$$
N_{m}(v)=\left[H_{m-n}(v) \sqrt{Q_{2 n}(v)}\right]_{+}=W_{m}^{\prime}(v) .
$$

The two equations (16) and (18) can be rephrased as $N_{m}=W_{m}^{\prime}$ and $\tilde{f}_{m-1}+N_{m}^{2}-Q_{2 n} H_{m-n}^{2}=0$ for some polynomial $\tilde{f}_{m-1}$ of order $m-1$, and $m$ can in general be different from $N-1$.

Summarizing, the world-volume of the M5-brane is $\mathbb{R}^{4} \times \Sigma$ where $\Sigma$ is a Riemann surface embedded in $\mathbb{R}^{5} \times S^{1}$ with complex coordinates $t, w, v$. The Riemann surface is defined by the following embedding:

$$
\begin{aligned}
t^{2}-2 P_{N}(v) t+\Lambda_{N=2}^{2 N} & =0 \\
w^{2}-2 W_{m}^{\prime}(v) w-\tilde{f}_{m-1}(v) & =0
\end{aligned}
$$


which indeed gives a one-dimensional complex surface. Notice that $\Sigma$ can be viewed as the intersection of two different Riemann surfaces, one in the $t-v$ plane defined by the first equation, and the other in the $w-v$ plane, defined by the second equation. This "doubling" precisely parallels the field theory behavior [15].

Furthermore, $\mathcal{N}=1$ supersymmetry imposes factorization formulas on this Riemann surface, so that its genus is reduced from $N-1$ to $n-1$ :

$$
\begin{aligned}
P_{N}^{2}(v)-\Lambda_{N=2}^{2 N} & =S_{N-n}^{2}(v) Q_{2 n}(v) \\
W_{m}^{\prime}(v)^{2}+\tilde{f}_{m-1} & =H_{m-n}^{2}(v) Q_{2 n}(v) .
\end{aligned}
$$

These are the requirements that there exist meromorphic functions on the Riemann surface with the prescribed boundary conditions.

So we see that, except at the points where $v$ blows up, one can think of $v$ and

$$
y=\sqrt{Q(v)}
$$

as coordinates on the Riemann surface, which has genus $n-1$, with branch cuts between the $2 n$ zeroes $q_{i}$ of $Q(v)$. We will follow the notation in [4], where a point is denoted by $p$ if $(v, y)=(p, \sqrt{Q(p)})$ and by $\tilde{p}$ if $(v, y)=(p,-\sqrt{Q(p)})$. We will also decompose $Q(v)$ as

$$
Q(v)=G_{n}^{2}(v)+f_{n-1}(v) .
$$

At this point it is clear that $t$ and $w$ have precisely the same properties as the gauge theory and matrix model resolvents $[1,15]$, where

$$
T(v)=\frac{\mathrm{d} t}{t}=\operatorname{Tr}_{\text {gauge theory }}\left(\frac{\mathrm{d} v}{v-\Phi}\right)=\mathrm{d} \log \left(P_{N}+y\right)
$$

is the gauge theory resolvent, whereas

$$
\begin{aligned}
R(v) & =w \mathrm{~d} v=2 \operatorname{Tr}_{\text {matrix theory }}\left(\frac{\mathrm{d} v}{v-M}\right)=-\frac{1}{16 \pi^{2}} \operatorname{Tr}_{\text {gauge theory }}\left(\frac{W_{\alpha} W^{\alpha} \mathrm{d} v}{v-\Phi}\right) \\
& =W_{m}^{\prime}(v)-\sqrt{W_{m}^{\prime}(v)^{2}+\tilde{f}_{m-1}(v)}
\end{aligned}
$$

is the matrix model resolvent. Notice that the two branches, classical and quantum, of the Riemann surface, arise naturally as the two asymptotic regions of the 5-brane.

So far the discussion was on-shell. Indeed, fixing the boundary conditions $W_{m}^{\prime}(v)$ at infinity completely fixes all the remaining coefficients, and so there is no room for holomorphic deformations of the brane configuration. In the next section we will see how to define deformations of $y$ so our discussion can parallel the deformations of the gauge theory where one turns on expectation values for the gluino condensates. We first discuss a few aspects of the twisted world-volume theory.

\subsection{The twisted $(2,0)$ theory}

The world-volume theory of M5-branes is quite complicated due to the presence of a self-dual tensor field. For general curved world-volumes embedded in eleven-dimensional supergravity, 
the world-volume theory for a single M5-brane was constructed in [18, 19]. A simple action for a flat M5-brane in flat eleven dimensions is given in [20], although this action also depends on the anti-self dual part of the tensor field. The latter paper also contains a useful summary of the various symmetries of the theory.

The standard $(2,0)$ theory has sixteen supercharges that transform as $(\mathbf{4}, \mathbf{4})$ under $S O(5,1) \times$ $S O(5)_{R}$, the product of the Lorentz group and the $S O(5)$ R-symmetry group. However, once we wrap the M5-brane on a non-trivial Riemann surface, the world-volume theory is automatically twisted [21]. The twist is such that the transverse scalars, which for a flat brane live in a trivial bundle over the world-volume, become sections of the normal bundle over the Riemann surface. To describe the twist, we notice that the Lorentz group is reduced from $S O(5,1)$ to $S O(3,1) \times S O(2)$ once we wrap the fivebrane on a non-trivial surface. Twisting the theory means that we embed the $S O(2)$ part of the broken Lorentz group in the $S O(5)$ R-symmetry group. Since one of the five scalars is always trivial $\left(x^{9}\right.$ in our notation), we need to embed $S O(2)$ in a $S O(4)=S U(2) \times S U(2)$ subgroup of $S O(5)_{R}$.

To determine the quantum numbers after the twisting, we first write the quantum numbers of the scalars and supercharges in terms of $S U(2) \times S U(2) \times U(1) \times S U(2)_{L} \times S U(2)_{R}$, where the first two $S U(2)$ 's represent the Lorentz group $S O(3,1)^{1}$, the $U(1) \sim S O(2)$ represents the internal part of the Lorentz group, and the last two $S U(2)$ 's represent the $S O(4) \subset S O(5)$ subgroup of the R-symmetry group that plays a role in the twisting. Before the twisting, the scalars and supercharges transform as

\begin{tabular}{|c|c|}
\hline$\Phi$ & {$\left[(\mathbf{1}, \mathbf{1})_{0},(\mathbf{2}, \mathbf{2})\right] \oplus\left[(\mathbf{1}, \mathbf{1})_{0},(\mathbf{1}, \mathbf{1})\right]$} \\
\hline$Q$ & {$\left[(\mathbf{2}, \mathbf{1})_{1},(\mathbf{2}, \mathbf{1})\right] \oplus\left[(\mathbf{2}, \mathbf{1})_{1},(\mathbf{1}, \mathbf{2})\right]$} \\
\hline $\bar{Q}$ & {$\left[(\mathbf{1}, \mathbf{2})_{-1},(\mathbf{2}, \mathbf{1})\right] \oplus\left[(\mathbf{1}, \mathbf{2})_{-1},(\mathbf{1}, \mathbf{2})\right]$} \\
\hline
\end{tabular}

where $\left[\left(\mathbf{j}_{\mathbf{1}}, \mathbf{j}_{\mathbf{2}}\right)_{m},\left(\mathbf{j}_{\mathbf{3}}, \mathbf{j}_{\mathbf{4}}\right)\right]$ contains the quantum numbers $\left(j_{1}, j_{2}, m, j_{3}, j_{4}\right)$ under $S U(2) \times S U(2) \times$ $U(1) \times S U(2)_{L} \times S U(2)_{R}$, the first two $S U(2)$ 's being the Lorentz group and the last two the R-symmetry.

The $U(1)$ that rotates $\log t$ but not $w$ is the $U(1)$ that is diagonally embedded in $S U(2)_{L} \times$ $S U(2)_{R}$, whereas the $U(1)$ that rotates $w$ but not $\log t$ is the off-diagonal one. Therefore, the $\mathcal{N}=2$ twist is the one where the $U(1)$ part of the Lorentz group is diagonally embedded in $S U(2)_{L} \times S U(2)_{R}$, whereas the $\mathcal{N}=1$ twist is the one where the $U(1)$ is embedded in $S U(2)_{L}$ only. After the $\mathcal{N}=2$ twist the fields transform as

\begin{tabular}{|c|c|}
\hline$\Phi$ & $(\mathbf{1}, \mathbf{1})_{0} \oplus(\mathbf{1}, \mathbf{1})_{0} \oplus(\mathbf{1}, \mathbf{1})_{0} \oplus(\mathbf{1}, \mathbf{1})_{2} \oplus(\mathbf{1}, \mathbf{1})_{-2}$ \\
\hline$Q$ & $(\mathbf{2}, \mathbf{1})_{2} \oplus(\mathbf{2}, \mathbf{1})_{2} \oplus(\mathbf{2}, \mathbf{1})_{0} \oplus(\mathbf{2}, \mathbf{1})_{0}$ \\
\hline $\bar{Q}$ & $(\mathbf{1}, \mathbf{2})_{0} \oplus(\mathbf{1}, \mathbf{2})_{0} \oplus(\mathbf{1}, \mathbf{2})_{-2} \oplus(\mathbf{1}, \mathbf{2})_{-2}$ \\
\hline
\end{tabular}

From this we see that half of the supersymmetries transform as a scalar on the Riemann surface, and these supersymmetries give rise to the unbroken $\mathcal{N}=2$ in four dimensions. In addition, $\log t$ transforms as a section of $\mathcal{O}(-2)$ and $w$ as a section of $\mathcal{O}(0)$. This is consistent with the fact that the normal bundle of the Riemann surface in $\mathbb{R}^{5} \times S^{1}$ should have degree -2 .

\footnotetext{
${ }^{1}$ Rather, $S U(2) \times S U(2)$ represents the Euclidean Lorentz group, but we trust that this will not cause any confusion.
} 
If we embed $U(1)$ in $S U(2)_{L}$, the scalar fields and supercharges transform as

\begin{tabular}{|c|c|}
\hline$\Phi$ & $(\mathbf{1}, \mathbf{1})_{0} \oplus(\mathbf{1}, \mathbf{1})_{1} \oplus(\mathbf{1}, \mathbf{1})_{1} \oplus(\mathbf{1}, \mathbf{1})_{-1} \oplus(\mathbf{1}, \mathbf{1})_{-1}$ \\
\hline$Q$ & $\left.(\mathbf{2}, \mathbf{1})_{2} \oplus(\mathbf{2}, \mathbf{1})_{1} \oplus \mathbf{2}, \mathbf{1}\right)_{1} \oplus(\mathbf{2}, \mathbf{1})_{0}$ \\
\hline $\bar{Q}$ & $(\mathbf{1}, \mathbf{2})_{0} \oplus(\mathbf{1}, \mathbf{2})_{-1} \oplus(\mathbf{1}, \mathbf{2})_{-1} \oplus(\mathbf{1}, \mathbf{2})_{-2}$ \\
\hline
\end{tabular}

Now both $\log t$ and $w$ transform as sections of $\mathcal{O}(-1)$. Only $1 / 4$ of the supersymmetries transform as a scalar, and these yield the surviving $\mathcal{N}=1$ generators in four dimensions. The R-symmetry of the resulting $\mathcal{N}=1$ field theory acts as (minus) the internal $U(1)$ piece of the Lorentz group before twisting. In particular, $w$ and $\log t$ have no $R$-charge, whereas the one-form $\mathrm{d} v$ has $\mathrm{R}$ charge 2, and $Q$ has R-charge -1 . In terms of the $S U(2) \times S U(2) \times U(1) \times U(1)_{L} \times U(1)_{R}$ subgroup of $S U(2) \times S U(2) \times U(1) \times S U(2)_{L} \times S U(2)_{R}$, the charges of $\log t, w$ and the unbroken $\mathcal{N}=1$ generator $Q_{\alpha}$ are

\begin{tabular}{|c|c|}
\hline $\log t$ & {$\left[(\mathbf{1}, \mathbf{1})_{0},(-1,-1)\right]$} \\
\hline$w$ & {$\left[(\mathbf{1}, \mathbf{1})_{0},(-1,+1)\right]$} \\
\hline$Q_{\alpha}$ & {$\left[(\mathbf{2}, \mathbf{1})_{1},(-1,0)\right]$} \\
\hline
\end{tabular}

We normalized the eigenvalues of $U(1)$ in such a way that they are always integer, i.e. they are twice the spin. We also pick one of the other three supersymmetry generators (which is broken once we wrap the five brane), denoted by $\hat{Q}_{\alpha}$, with quantum numbers $\left[(\mathbf{2}, \mathbf{1})_{1},(0,-1)\right]$. Though the corresponding symmetry is broken, we can still use it to build a supermultiplet, which we write in terms of an auxiliary superspace with anti-commuting coordinates $\eta^{\alpha}$. Using the quantum numbers of $\hat{Q}_{\alpha}$ we find that an example of such a multiplet is

$$
F=w+\chi_{\alpha} \eta^{\alpha}+\frac{\partial \log t}{\partial v} \eta^{\alpha} \eta_{\alpha}
$$

with some anti-commuting degree of freedom $\chi_{\alpha}$. if we compute the A-periods (4) of the differential $F \mathrm{~d} v$, we obtain a four-dimensional supermultiplet of the form

$$
\frac{1}{2 \pi i} \oint_{A_{i}} F \mathrm{~d} v=S_{i}+w_{\alpha} \eta^{\alpha}+N_{i} \eta^{\alpha} \eta_{\alpha}
$$

which has precisely the form of the auxiliary supermultiplet introduced in [15]. The full effective action can be written as an integral over auxiliary superspace of a holomorphic function of this auxiliary supermultiplet. Thus, this auxiliary structure seems to have a direct origin in the supersymmetry of the M5-brane. It would be interesting to work out the twist and the corresponding KK reduction in more detail, as this would no doubt shed further light on the structure of the four-dimensional effective action.

\section{The off-shell superpotential}

In [5], Witten defined a superpotential in terms of the M5-brane coordinates that reproduces the on-shell value of the effective superpotential in the gauge theory. Holomorphic configurations are minima of this superpotential.

The aim of this section is to show that this superpotential not only gives the correct on-shell result, but can also be evaluated off-shell, and its off-shell value reproduces the Dijkgraaf-Vafa result in terms of the gluino condensates. 
The precise definition of the superpotential is actually quite subtle, because the Riemann surface we are considering is non-compact. However, before we turn to a discussion of the superpotential we first need to describe the off-shell geometry of the M5-brane.

\subsection{Off-shell deformations of the M5-brane}

The on-shell configuration for the five-brane, as given in equations (19), (20) and (22), reads

$$
\begin{aligned}
t^{2}-2 P_{N}(v) t+\Lambda_{N=2}^{2 N} & =0 \\
w^{2}-2 W_{m}^{\prime}(v) w-\tilde{f}_{m-1}(v) & =0,
\end{aligned}
$$

where

$$
\begin{aligned}
P_{N}^{2}-\Lambda^{2 N} & =S_{N-n}^{2}\left(G_{n}^{2}+f_{n-1}\right) \\
\left(W_{m}^{\prime}\right)^{2}+\tilde{f}_{m-1} & =H_{m-n}^{2}\left(G_{n}^{2}+f_{n-1}\right) .
\end{aligned}
$$

Both $t$ and $w$ can be viewed as functions on the underlying Riemann surface $y^{2}=G_{n}^{2}+f_{n-1}$. We are interested in varying this underlying Riemann surface, so that the glueball degrees of freedom $S_{i} \sim \operatorname{Tr}_{U\left(N_{i}\right)}\left(\mathcal{W}^{2}\right)$ acquire a non-trivial expectation value. According to (4), this is the same as changing the $A$-periods of the differential $w \mathrm{~d} v$, which can be accomplished by varying the function $f_{n-1}$. However, we should also specify the embedding of this new Riemann surface in $\mathbb{R}^{5} \times S^{1}$, i.e. give new functions $t$ and $w$ on this Riemann surface. Since the glueball fields $S_{i}$ are chiral superfields, one might be inclined to believe that the relevant deformations of $t$ and $w$ should be holomorphic. As we will now argue, this turns out to be incorrect.

If $t$ and $w$ were still holomorphic after the deformation, one might believe that they would still obey equations of the form (32) and (33). A simple count of the number of free parameters shows that this cannot work. Once we specify $W_{m}^{\prime}$ and the $n A$-periods of $w \mathrm{~d} v$, which we trade off for the coefficients in $f_{n-1}$, the number of free parameters remaining in equations (32) and (33) is $N+(N-n)+n+m+m-n=2 N+2 m-n$. The number of equations is $2 N+2 m$, which is larger than the number of free parameters, and the system will generically have no solution.

Of course, there could exist holomorphic deformations that don't preserve the factorized form (32) and (33). A naive counting argument shows that this is unlikely as well. If we consider a compact Riemann surface $\Sigma$ of genus $g$ embedded in a Calabi-Yau of complex dimension $d$, then a straightforward application of the index theorem yields that $\operatorname{dim} H^{0}(\Sigma, \mathcal{N})-\operatorname{dim} H^{1}(\Sigma, \mathcal{N})=$ $(d-3)(1-g)$, with $\mathcal{N}$ the normal bundle, so as long as $\operatorname{dim} H^{1}(\Sigma, \mathcal{N})=0$ the number of holomorphic sections of the normal bundle is simply $(d-3)(1-g)$. This is quite naive for many reasons, first of all in our case the Riemann surface is noncompact, there may in general be obstructions to lift a holomorphic section of the normal bundle to a finite holomorphic deformation, and $H^{1}$ may be nonzero. Nevertheless, if we apply this result to the present case, we see that there are no holomorphic deformations, as $d=3$.

If we ignore the coordinate $t$ for the moment, then a count of parameters in equation (33) shows that this equation does generically have a solution for arbitrary $f_{n-1}$. Thus, there is no problem defining a holomorphic function $w$ on the Riemann surface, all problems are caused by the coordinate $t$. 
To resolve this issue, it is helpful to slightly change perspective and to think of the one-forms $w \mathrm{~d} v$ and $\mathrm{d} t / t$ instead of the functions $w$ and $t$. These have an interpretation as matrix model and gauge theory resolvents as in equations (5) and (6), and it should be possible to determine them off-shell. This was indeed done in [4]. Off-shell, $w \mathrm{~d} v$ is some differential with periods around the cycles of the Riemann surface that are directly related to the glueball vevs. Therefore, we expect that once we go off-shell, the relation (33) is still satisfied, with $f_{n-1}$ parametrizing the deformations. In other words, in the $v, w$ plane going off-shell simply corresponds to making a holomorphic change of the Riemann surface.

The situation regarding $t$ is more complicated. In a vacuum of the theory, the holomorphic one-form $\mathrm{d} t / t$ has integer periods around all compact cycles of the Riemann surface. The fact that the periods are integer means that $\log t$ is a well-defined function with values in $\mathbb{R} \times S^{1}$ on the Riemann surface (or put differently, this follows from the fact that $H^{0}\left(\Sigma, \mathbb{C}^{*}\right) \equiv H^{1}(\Sigma, \mathbf{Z})$ ). Once we go off-shell by a geometric deformation in the $w, v$ plane, there no longer is a holomorphic one-form with integer periods around all cycles. However, there is a non-holomorphic closed one-form, which is defined in such a way that it has the same integer periods around both the A and $\mathrm{B}$ cycles as $\mathrm{d} t / t$ had in the vacuum of the theory, and the same pole structure at infinity. We will continue to call this one-form $\mathrm{d} t / t$. It is indeed appropriate to call the form $\mathrm{d} t / t$, because there still will be a global $\mathbb{R} \times S^{1}$ valued function $\log t$ associated to $\mathrm{d} t / t$, and we will take this function to define the embedding of the Riemann surface in the $t$-direction. However, this embedding will no longer be holomorphic.

To summarize: to go off-shell we need to perform a holomorphic deformation in the $w, v$ plane, and a non-holomorphic deformation in the $t$-direction, defined by a non-holomorphic one-form with integer periods.

The non-holomorphic one-form $\mathrm{d} t / t$ is denoted by $T_{0}$ in [4]. It is off-shell no longer equal to the resolvent of the gauge theory. The latter is given by a holomorphic one-form $T$, which has the same periods around the A-cycles as $T_{0}$, but non-integer B-periods. On-shell, $T$ and $T_{0}$ coincide. We will discuss the fivebrane interpretation of $T$ in section 6 .

Another perspective on the five-brane is that it represents a pure geometrical dual of the setup of [2]. In this setup one considers a noncompact Calabi-Yau with either wrapped branes or with a non-trivial three-form flux with integer periods. Quantities computed using this non-compact Calabi-Yau can be computed in terms of a Riemann surface. The three-form flux reduces to a one-form on this Riemann surface with integral periods. This is exactly the same one-form as $\mathrm{d} t / t$. Therefore we see that after dualizing to M-theory, the flux becomes purely geometrical and encodes the embedding of the fivebrane in the ambient space in the $t$-direction. The periods of the flux become the winding numbers of the $\mathbb{C}^{*}$ variable $t$ around the corresponding cycles.

\subsection{Computing the superpotential}

Now that we have learned how to deform the Riemann surface, we would like to consider the off-shell computation of the superpotential for the fivebrane configuration, leaving a detailed analysis of the non-holomorphic embedding $t$ for the next section. Obviously, we expect to recover the standard expression for the superpotential, but we would like to see how this comes about.

We will use the notation in [5], where one thinks of $\Sigma$ as a surface with a map $\Phi: \Sigma \rightarrow X$. 
We will take this to be an embedding, although our considerations should generalize as in [5]. This map is described by functions $\phi^{i}(\lambda)$, where $\lambda$ are coordinates on $\Sigma$.

The expression that Witten postulated in [5] for the superpotential for M5 branes wrapping a two-cycle is

$$
W(\Sigma)-W\left(\Sigma_{0}\right)=\frac{1}{2 \pi i} \int_{B} \Omega
$$

where $B$ is a 3 -chain interpolating between $\Sigma$ and $\Sigma_{0}$, and $\Sigma_{0}$ is an arbitrary reference surface in the same homology class as $\Sigma$. In our coordinates, the holomorphic 3 -form $\Omega$ is $\Omega=\Omega_{i j k} \mathrm{~d} \phi^{i} \wedge$ $\mathrm{d} \phi^{j} \wedge \mathrm{d} \phi^{k}=R \mathrm{~d} v \wedge \mathrm{d} w \wedge \mathrm{d} t / t$. As a basis for one-forms $\mathrm{d} \phi^{i}$ we will use $\mathrm{d} v, \mathrm{~d} w, \mathrm{~d} \log t$.

We will choose coordinates such that $v$ is a good function on either sheet of the Riemann surface, except at infinity where it has a pole. Minimizing the superpotential then amounts to finding suitable meromorphic functions $(t, w)$ on this surface. Notice that from the 5 -brane perspective there is nothing particular about this choice, and one could just as well interchange the roles of $v$ and $w$.

Before proceeding, we point out that the definition of the superpotential in (34) requires $\Sigma_{0}$ and $\Sigma$ to have the same asymptotics, otherwise a certain regularization at infinity is required. In particular, this implies that (34) can only be used to study the variation under normalizable deformations of the curve (though we will also allow log-normalizable deformations in the remainder, but the final expression for the superpotential will be finite when we send the cutoff to infinity even off-shell). Of course, we are also interested in the variation of the superpotential under non-normalizable deformations of the curve, such as those associated to variations of the coefficients in the superpotential. To determine this variation, we need to supplement (34) with some additional information, otherwise we could choose arbitrarily different $\Sigma_{0}$ for each non-normalizable deformation and the result for the superpotential would be arbitrary.

Note also that if $\Sigma$ were compact, the superpotential would be zero for any holomorphic $\Sigma$. The fact that we get a non-trivial superpotential as a function of holomorphic variables is due to the non-compactness of $\Sigma$. (Another way in which this can happen is if there is a global obstruction to certain holomorphic deformations, see e.g. [22]).

We now first consider the extrema of the superpotential (34). If we perform a generic variation of the superpotential we obtain

$$
2 \pi i \delta W=3 \int_{\Sigma} \Omega_{i j k} \delta \phi^{i} \mathrm{~d} \phi^{j} \wedge \mathrm{d} \phi^{k}=0
$$

which we rewrite as

$$
\int_{\Sigma} \Omega_{i j} \delta \phi^{i} \wedge \mathrm{d} \phi^{j}=0
$$

where $\Omega_{i j}$ is a reduced 1-form on the Riemann surface, $\Omega_{i j}=\Omega_{i j v} \mathrm{~d} v$, and $i$ runs over $(\log t, w)$.

On $\Sigma-\{P, Q\}$, this implies that $w$ and $t$ should holomorphic functions of $v$. This is already enough to find the form of $w$ and $t$, once we impose suitable boundary conditions on $w$ and $t$, and assume the underlying Riemann surface is hyperelliptic.

To see this, we notice that any holomorphic function $u$ on the hyperelliptic Riemann surface $y^{2}=G_{n}^{2}(v)+f_{n-1}(v)$ can be written as $u=A(v)+y B(v)$, with $A$ and $B$ meromorphic functions of $v$. Clearly, $u$ satisfies

$$
(u-A(v))^{2}=B^{2}(v)\left(G_{n}^{2}(v)+f_{n-1}(v)\right)
$$


and after multiplication with a suitable polynomial we see that $u$ must satisfy an equation of the form

$$
C(v) u^{2}+D(v) u+E(v)=0
$$

with polynomials $C, D, E$ with no common factors. In addition, there must exist a polynomial $F$ so that

$$
D^{2}(v)-4 C(v) E(v)=F^{2}(v)\left(G_{n}^{2}(v)+f_{n-1}(v)\right) .
$$

If $C(v)$ has a zero for some finite value of $v$, then at that value of $v$ at least one of the two solutions $u$ of (38) would go to infinity, which contradicts the boundary condition that $u$ should be holomorphic in $\Sigma-\{P, Q\}$. Therefore, $C(v)$ has to be a constant and can be taken equal to one. The boundary conditions on $t$ and $w$ together with the constraint (39) then completely fix the form of the remaining polynomials $D(v)$ and $E(v)$ for both $t$ and $w$. Notice that $t$ should not be zero anywhere on $\Sigma-\{P, Q\}$ and therefore one immediately sees that for $t$ the polynomial $E$ should be a constant. A zero of $t$ is not acceptable because $t$ was related to $x^{6}$ and $x^{10}$ by $t=\exp \left(-R^{-1}\left(x^{6}+i x^{10}\right)\right)$.

The final result for $t$ and $w$ is summarized in (19), (32) and (33).

To obtain the superpotential as a function of glueball superfield vacuum expectation values, we should give an expression for it which is valid off-shell, i.e. once we turn on the deformations discussed in section 3.1.

After a partial integration, and after dropping the contribution from $\Sigma_{0}$ which is just a constant, we obtain

$$
W_{\text {eff }}=\frac{R}{2 \pi i} \int_{\Sigma} \frac{\mathrm{d} t}{t} \wedge w \mathrm{~d} v=\frac{R}{2 \pi i} \int_{\Sigma} \eta^{1} \wedge \eta^{2}
$$

where we defined the two one-forms

$$
\eta^{1}=w \mathrm{~d} v, \quad \eta^{2}=\frac{\mathrm{d} t}{t}
$$

It is important to pause for a moment here to discuss the partial integration. In general, on a non-compact Riemann surface, one has to determine what one keeps fixed at infinity (see e.g. the discussion in [23] where the variable describing the size of disc instantons was kept fixed). Here, what we would like to keep fixed is the coefficients in the tree-level superpotential, which determine the asymptotics of $w$. Thus, we keep $w$ fixed at infinity, and that is why we extract $w$ in (40), rather then e.g. $\log t$.

The deformations can be parametrized by the coefficients in $f_{n-1}(v)$, that appears in the defining equation

$$
y^{2}=G_{n}^{2}(v)+f_{n-1}(v)
$$

for the Riemann surface. The polynomial $f_{n-1}(v)$ can be expanded as

$$
f_{n-1}(v)=\sum_{i=0}^{n-1} f_{i} v^{i}
$$

where the coefficients $f_{i}$ with $i=0, \ldots, n-2$ correspond to normalizable deformations, and the coefficient $f_{n-1}$ to a log-normalizable deformation ${ }^{2}$. Equivalently, we can use the A-periods of

\footnotetext{
${ }^{2}$ We use the same symbol for the polynomial $f_{n-1}(v)$ and its coefficients, $f_{i}$, but hopefully this will not cause any confusion.
} 
$\eta^{1}=w \mathrm{~d} v$ to parametrize the deformation,

$$
S_{i}=\frac{1}{2 \pi i} \oint_{A_{i}} w \mathrm{~d} v
$$

where as usual the A-cycles are compact and run around the branch cuts of $y$ in the $v$-plane as determined by the hyperelliptic form $y^{2}=G_{n}^{2}+f_{n-1}$ of $\Sigma$, see appendix A. The A-periods of $\eta^{2}=\mathrm{d} t / t$ are also integers,

$$
2 \pi i N_{i}=\oint_{A_{i}} \frac{\mathrm{d} t}{t}
$$

which are the ranks of the gauge groups that remain classically unbroken in a particular classical minimum of the superpotential. This can easily be seen by taking the classical limit $\Lambda_{N=2} \rightarrow 0$ of the M5 brane configuration. In that case,

$$
P_{N}(v) \rightarrow \prod_{i=1}^{n}\left(v-c_{i}\right)^{N_{i}}
$$

and so the periods are equal to $2 \pi i N_{i}$.

The A-cycles are dual to noncompact B-cycles. We know from the special geometry relations of Calabi-Yau manifolds that

$$
\frac{\partial \mathcal{F}_{0}}{\partial S_{i}}=\frac{1}{2 \pi i} \oint_{B_{i}} w \mathrm{~d} v
$$

where $\mathcal{F}_{0}$ is the prepotential. If we in addition for the moment assume that the integer periods of $\mathrm{d} t / t$ around the compact B-cycles all vanish, then the integrals of $\mathrm{d} t / t$ along all non-compact B-cycles are all identical,

$$
\begin{aligned}
2 \pi i \tau & =\int_{B_{n}} \frac{\mathrm{d} t}{t} \\
0 & =\oint_{B_{i}} \frac{\mathrm{d} t}{t},
\end{aligned}
$$

$i=1, \ldots, n-1$, where $\tau$ will have the interpretation as the gauge coupling.

At this point we can invoke the Riemann bilinear identities, and rewrite (40) as

$$
W_{\text {eff }}=R \sum_{i=1}^{n}\left(N_{i} \frac{\partial \mathcal{F}_{0}}{\partial S_{i}}-2 \pi i \tau S_{i}\right)=R \sum_{i=1}^{n} N_{i} \frac{\partial \mathcal{F}_{0}}{\partial S_{i}}-R N S \log \frac{\Lambda_{N=2}}{\Lambda_{0}},
$$

which is precisely the Dijkgraaf-Vafa answer. The scale $\Lambda_{0}$ appears because the integral of $\mathrm{d} t / t$ around non-compact cycles needs to be regularized, see appendix A.

Actually, once we accept (40) and once we identify $\mathrm{d} t / t$ with the one-form that one obtains by reducing the three-form flux on the dual type IIB side, we see that (40) is nothing but the reduction of the type IIB superpotential $\int H \wedge \Omega$ to the Riemann surface. The minimalization of (49) from this point of view has already been discussed in [24]. They showed that at the minimum of (49) there exists a meromorphic function with an $N$-th order pole at $\tilde{P}$ and an $N$ th order zero at $P$. Of course, this meromorphic function is nothing but $t$, and at the minimum we recover the holomorphic fivebrane configuration of M-theory.

This discussion is somewhat imprecise, because (40) is still not quite well-defined as it stands, and because there is in principle no reason why the periods of $\mathrm{d} t / t$ around the compact B-cycles 
should vanish. A more precise discussion and the interpretation of the periods of $\mathrm{d} t / t$ around the B-cycles is given in [3, 4], see in particular section 7.1 of [4]. There, a regulated version of (40) is defined; as it stands, (40) is problematic because both $\eta^{1}$ and $\eta^{2}$ have poles at $P, \tilde{P}$. To make it well-defined, one moves the poles of $\eta^{1}=\mathrm{d} t / t$ to nearby points $P^{\prime}, \tilde{P}^{\prime}$. Next one applies a suitable version of the Riemann bilinear identities for closed differentials with poles, of the type discussed in section III.3 of [25]. This then gives a precise regulated definition of (40), and one can again show quite generally that at the extremum $\mathrm{d} t / t$ becomes a meromorphic differential. The only effect of the periods, $\tau_{i}$, of $\mathrm{d} t / t$ around the compact B-cycles is an extra term

$$
W_{\mathrm{eff}}^{\prime}=2 \pi i R \sum_{i=1}^{n-1} \tau_{i} S_{i}
$$

in (49).

To see more directly that on-shell $\mathrm{d} t / t$ is the gauge theory resolvent we manipulate the expression in (40) as follows. We first write it as

$$
\frac{R}{2 \pi i} \int_{\Sigma} w \bar{\partial} \log t \mathrm{~d} v \mathrm{~d} \bar{v}
$$

Naively, this expression vanishes, as on-shell $\log t$ is a holomorphic function of $v$. However, one has to be careful, as $t$ has poles at infinity. We can rewrite this expression as follows:

$$
\begin{aligned}
W_{\text {eff }} & =-\frac{R}{2 \pi i} \int_{\Sigma} \mathrm{d}(w \log t \mathrm{~d} v)=-\frac{R}{2 \pi i} \oint_{P} w \log t \mathrm{~d} v \\
& =-2 R \operatorname{Res}_{v=\infty}\left(\log t\left(W_{m}^{\prime}(v)+\text { subleading }\right)\right) \\
& =2 R \operatorname{Res}_{v=\infty}\left(\frac{\partial \log t}{\partial v} W_{m}(v)\right)
\end{aligned}
$$

where we assume that $P$ is the point where $t \sim v^{N}, w \sim 2 W_{m}^{\prime}(v)$. In order for this to be the right answer, we need that $\mathrm{d} \log t$ is proportional to the gauge theory resolvent, see also (23), so that this equation reduces to the classical superpotential evaluated at the right quantum vacuum.

Thus we see that these two ways to interpret (40) give two equivalent answers for the superpotential, one off-shell as in Dijkgraaf-Vafa, the other directly on-shell in terms of the gauge theory resolvent. Interestingly, the computations appearing here bear a close resemblance to those that appear in the study $[26,27]$ of the integrable models that are needed to write down the superpotentials of these theories once they are compactified on $S^{1}$. At this point this seems somewhat coincidental, as the degrees of freedom describe off-shell deformations are quite different depending on whether one compactifies the theory on a circle or not.

\section{Off-shell embedding of the fivebrane}

We have seen that in order to go off-shell we need to embed the M5-brane non-holomorphically in $\mathbb{R}^{5} \times S^{1}$. The coordinate $w$ remains a holomorphic function of $v$ and is parametrized by the $n$ glueball vevs $S_{i}$, or equivalently by the coefficients of $f_{n-1}(v)$. The coordinate $t$ on the other 
hand becomes non-holomorphic, $t=t(v, \bar{v})$, so that the periods

$$
\begin{aligned}
& \oint_{A_{i}} T=2 \pi i N_{i} \\
& \oint_{B_{i}} T=2 \pi i \tau_{i}
\end{aligned}
$$

Our goal in this section is to study in some more detail the form of $T$ (or equivalently $t$ ) implied by (53).

\subsection{General procedure}

The general idea to solve for (53) is to write $T$ as a linear combinations of closed one-forms. The number of one-forms compatible with the asymptotic behavior of $T$ is $2 n$, so that the $2 n$ equations in (53) indeed completely determine $T$. There are two different convenient bases of one-forms that we will use. The first one, which we will call the orthonormal basis, is useful for many purposes since their $A$-periods are canonically normalized [25]. The second one, which we will call a basis of monomials, is a more natural basis if we are interested in explicitly integrating $T$ and extracting the embedding coordinate $t$.

\subsubsection{Orthonormal basis}

As told, $T(v, \bar{v})=\frac{\mathrm{d} t}{t}$ is off-shell a generic closed one-form with fixed periods (53). Expanding upon a basis of holomorphic one-forms, we can write it as

$$
T(v)=a \tau_{P, \tilde{P}}+b \bar{\tau}_{P, \tilde{P}}+\sum_{i=1}^{n-1}\left(h_{i} \xi_{i}+l_{i} \bar{\xi}_{i}\right)
$$

where $\xi_{i}$ is a basis of holomorphic differentials $\left\{\xi_{1}, \ldots, \xi_{n-1}\right\}$ normalized such that $\oint_{A_{i}} \xi_{j}=\delta_{i j}$, and which vanish at infinity. In addition, the A-periods of $\tau_{P, \tilde{P}}$ are chosen to vanish, and its residue at $P, \tilde{P}$ are 1 and -1 , respectively. For a particular definition of the periods, see appendix A.

The differentials $\xi_{i}$ are all of the form $\frac{u_{i}(v)}{y} \mathrm{~d} v$ for some polynomials $u_{i}(v)$ of order at most $n-2$. The differential $\tau_{P, \tilde{P}}$ is of the form $\frac{u_{n-1}(v)}{y} \mathrm{~d} v$ with $u_{n-1}(v)$ a polynomial of order $n-1$ with leading coefficient one. In the monomial basis we will use the differential forms $\frac{v^{i}}{y} \mathrm{~d} v$ instead.

Inserting (54) in (53) leads to a set of equations for $a, b, h_{i}, l_{i}$. In this computation, one has to bear in mind that the integral over the non-compact period $B_{n}$ is defined as before, by conveniently regularizing the period with $\Lambda_{0}$. We find:

$$
\begin{aligned}
N & =a-b \\
2 \pi i N_{i} & =h_{i}-l_{i} \\
2 \pi i \tau_{i} & =\sum_{j=1}^{n-1}\left(h_{i} \Pi_{i j}-l_{i} \bar{\Pi}_{i j}\right)+2 \pi i a \int_{\tilde{\Lambda}_{0}}^{\Lambda_{0}} \xi_{i}-2 \pi i b \int_{\tilde{\Lambda}_{0}}^{\Lambda_{0}} \bar{\xi}_{i} \\
2 \pi i \tau & =a \int_{\tilde{\Lambda}_{0}}^{\Lambda_{0}} \tau_{P, \tilde{P}}+b \int_{\tilde{\Lambda}_{0}}^{\Lambda_{0}} \bar{\tau}_{P, \tilde{P}}+\sum_{i=1}^{n-1}\left(h_{i} \int_{\tilde{\Lambda}_{0}}^{\Lambda_{0}} \xi_{i}+l_{i} \int_{\tilde{\Lambda}_{0}}^{\Lambda_{0}} \bar{\xi}_{i}\right)
\end{aligned}
$$


where the last integral is the integral over the non-compact period, and we used that (see e.g. $[4])$

$$
\frac{1}{2 \pi i} \oint_{B_{i}} \tau_{P, \tilde{P}}=\int_{\tilde{P}}^{P} \xi_{i}
$$

The above integrals are not easily computed in general, however, the leading $\Lambda_{0}$-dependence is easy to extract. The integrals $\int_{\tilde{\Lambda}_{0}}^{\Lambda_{0}} \xi_{i}$ and $\int_{\tilde{\Lambda}_{0}}^{\Lambda_{0}} \bar{\xi}_{i}$ only give finite contributions as $\Lambda_{0} \rightarrow \infty$. The large $\Lambda_{0}$ behavior of $\int_{\tilde{\Lambda}_{0}}^{\Lambda_{0}} \tau_{P, \tilde{P}}$ is

$$
\int_{\tilde{\Lambda}_{0}}^{\Lambda_{0}} \tau_{P, \tilde{P}}=\frac{2}{n+1} \log \left(\frac{-4 G_{n}^{2}\left(\Lambda_{0}\right)}{f_{n-1}\left(\Lambda_{0}\right)}\right)+\mathcal{O}\left(\frac{\log \Lambda_{0}}{\Lambda_{0}}\right)
$$

and therefore only involves the leading coefficients of the polynomials $G_{n}(v)$ and $f_{n-1}(v)$ that appear in the defining equation $y^{2}=G_{n}^{2}+f_{n-1}$ of the Riemann surface ${ }^{3}$. The only $\Lambda_{0}$-divergence in (55) is hence in the last equation

$$
2 \pi i \tau=a \log \Lambda_{0}^{2}+b \log \bar{\Lambda}_{0}^{2}+\text { const }+\mathcal{O}\left(\log \Lambda_{0} / \Lambda_{0}\right)
$$

On-shell, this equation is directly related to the running of the bare Yang-Mills coupling as a function of the field theory UV cutoff: $b=0$ and $a=N$ is proportional to the one-loop beta function of the field theory. The on-shell value of (57) can easily be computed using $G_{n}=P_{N}$ and $f_{n-1}=-\Lambda_{N=2}^{2 N}$, which yields $2 \pi i \tau=\log \left(-4 \Lambda_{0}^{2 N} / \Lambda_{N=2}^{2 N}\right)$. However, in order to obtain the usual $\mathcal{N}=1$ answers, we will make a slightly different choice, namely

$$
2 \pi i \tau=N \log \left(\frac{-2 \Lambda_{0}^{2}}{\Lambda_{N=2}^{2}}\right)
$$

which would correspond to an embedding of the form $t^{2}-P_{N}(v) t+\left(-\Lambda_{N=2}^{2} / 2\right)^{N}$. This change is just a change of normalization, and from here on we will use the identification (59), which also holds off-shell. If we combine (58) with (59) and with the equation $a-b=N$ of (55), we obtain

$$
-N \log \Lambda_{N=2}^{2}=b \log \left(\Lambda_{0}^{2} \bar{\Lambda}_{0}^{2}\right)+\text { const. }
$$

This shows that $b \rightarrow 0$ as $\Lambda_{0} \rightarrow \infty$. In other words, the Riemann surface has a non-holomorphic "tail", which is relevant at the scale of the cutoff $\Lambda_{0}$. As we send the cutoff to infinity, the non-holomorphic tail is sent to infinity as well, but it does yield a finite contribution to physical quantities for all values of the cutoff. Thus we should first compute the superpotential and then send the cutoff to infinity, and not the other way around. Although $b$ vanishes when the cutoff is taken to infinity, the Riemann surface will in general remain non-homolomorphic in the limit: as one can see from (55), the $l_{i}$ will in general remain non-zero.

It is interesting to see how the remaining non-holomorphic deformations, parametrized by $l_{i}$, disappear on-shell. We have already found that on-shell $b=0$. Now, on-shell we also have

$$
\tau_{i}=\sum_{j=1}^{n-1} N_{j} \Pi_{i j}+N \int_{\tilde{\Lambda}_{0}}^{\Lambda_{0}} \xi_{i}=\sum_{j=1}^{n} N_{j} \int_{\hat{B}_{j}} \xi_{i}
$$

\footnotetext{
${ }^{3}$ This assumes that the leading coefficient of $f_{n-1}$ is nonvanishing. If it vanishes, and the leading behavior of $f_{n-1}$ is $v^{m}$, then the same expression remains valid except that the prefactor should be changed to $2 /(2 n-m)$.
} 
where $\hat{B}_{j}$ are the non-compact periods, $\hat{B}_{i}=B_{i}+B_{n}$ for $i=1, \ldots, n-1$, and we used the manipulations in [15]. Here we regard $S_{i}$ as the on-shell expectation value of the gluino condensate. Combining this with equation (55), we get

$$
\sum_{j=1}^{n-1} l_{j} \operatorname{Im} \Pi_{i j}=0 .
$$

Notice that by construction $\operatorname{Im} \Pi_{i j}$ is positive definite. So it has no zero eigenvalues unless $l_{i}=0$, which is precisely the condition that all remaining non-holomorphic deformations vanish.

\subsubsection{Monomial basis}

The above expansion of the one-form $\mathrm{d} t / t$ in terms of canonically normalized one-forms on the Riemann surface allows us to write its periods in terms of the period matrix of the Riemann surface. However, if we want to write down an explicit expression for $t$, it is easier to expand it in the monomial basis

$$
\zeta_{i}=\frac{v^{i-1}}{y} \mathrm{~d} v, \quad 0 \leq i \leq n-1 .
$$

The differentials $\tau_{P, \tilde{P}}$ and $\xi_{i}$ can be written as linear combinations of the $\zeta_{i}$, and the nonnormalizable one-form $\zeta_{n-1}$ appears only in $\tau_{P, \tilde{P}}$ but not in the normalizable one-forms $\xi_{i}$. Thus, the one-form $\mathrm{d} t / t$ is of the form

$$
\frac{\mathrm{d} t}{t}=T(v, \bar{v})=\frac{a_{n-1}(v)}{y(v)} \mathrm{d} v+\frac{b_{n-1}(\bar{v})}{\bar{y}(\bar{v})} \mathrm{d} \bar{v}
$$

where $a_{n-1}(v)$ and $b_{n-1}(\bar{v})$ are polynomials in $v$ that are determined by the conditions on the periods. We will compute them in some explicit examples below.

It is straightforward to formally integrate $t$ from the expression for $T(v, \bar{v})$. We get:

$$
t(v, \bar{v})=\alpha(v) \beta(\bar{v})
$$

where $\alpha$ and $\beta$ satisfy

$$
\begin{aligned}
\frac{\alpha^{\prime}(v)}{\alpha(v)} & =\frac{a_{n-1}(v)}{y(v)} \\
\frac{\beta^{\prime}(\bar{v})}{\beta(\bar{v})} & =\frac{b_{n-1}(\bar{v})}{\bar{y}(\bar{v})}
\end{aligned}
$$

and are formally given by

$$
\begin{aligned}
& \alpha(v)=\exp \int \frac{a_{n-1}(v)}{y(v)} \mathrm{d} v \\
& \beta(\bar{v})=\exp \int \frac{b_{n-1}(\bar{v})}{\bar{y}(\bar{v})} \mathrm{d} \bar{v} .
\end{aligned}
$$

There is however an important restriction on $\alpha$ and $\beta$, which comes from the requirement that $t$ must be a well-defined function of $v$ and $\bar{v}$. This means that the product $\alpha(v) \beta(\bar{v})$ must be a well-defined function on the Riemann surface. This is a non-trivial requirement, as the formal integrals (67) are not necessarily smooth single-valued functions for all values of $v$, and in general they will not be. However, the products must be such that $t$ itself is well-defined as an embedding coordinate. Of course, this is equivalent to imposing that $T$ has integer periods. 


\subsection{Example}

To illustrate the above formalism, we will now apply it to the case where the gauge group is classically unbroken, so that it confines completely in the quantum theory. In this case the Riemann surface has a single cut and two punctures.

The Riemann surface is given by the equation

$$
y=\sqrt{(v-c)^{2}-\mu}
$$

The differential $\mathrm{d} t / t$ is therefore of the form

$$
\frac{\mathrm{d} t}{t}=\frac{a}{y} \mathrm{~d} v+\frac{b}{\bar{y}} \mathrm{~d} \bar{v}
$$

which can be integrated to give the explicit expression for $t(v, \bar{v})$

$$
t(v, \bar{v})=(v-c+y)^{a}(\bar{v}-\bar{c}+\bar{y})^{b} .
$$

We can compute the A-period and the regularized B-period of $\mathrm{d} t / t$, and this leads (see also (55)) for the A-period to

$$
\frac{1}{2 \pi i} \oint_{A} \frac{\mathrm{d} t}{t}=a-b=N
$$

and for the non-compact B-period we get

$$
2 \pi i \tau=\int_{B} \frac{\mathrm{d} t}{t}=\log \left(\frac{\Lambda_{0}-c+y\left(\Lambda_{0}\right)}{\Lambda_{0}-c-y\left(\Lambda_{0}\right)}\right)^{a}\left(\frac{\bar{\Lambda}_{0}-\bar{c}+\bar{y}\left(\bar{\Lambda}_{0}\right)}{\bar{\Lambda}_{0}-\bar{c}-\bar{y}\left(\bar{\Lambda}_{0}\right)}\right)^{b} .
$$

For large $\Lambda_{0}$, this becomes

$$
2 \pi i \tau=\log \left(\frac{4 \Lambda_{0}^{2}}{\mu}\right)^{a}\left(\frac{4 \bar{\Lambda}_{0}^{2}}{\bar{\mu}}\right)^{b}+\mathcal{O}\left(\log \Lambda_{0} / \Lambda_{0}\right)
$$

For simplicity we will now also assume that the superpotential is quadratic,

$$
W(\Phi)=\frac{1}{2} g_{2}(\Phi-c)^{2},
$$

so that the differential $w \mathrm{~d} v$ becomes

$$
w \mathrm{~d} v=g_{2}(v-c+y) \mathrm{d} v
$$

The periods are

$$
S=\frac{1}{2 \pi i} \oint_{A} w \mathrm{~d} v=-\frac{g_{2} \mu}{2}
$$

and

$$
\begin{aligned}
\frac{\partial \mathcal{F}}{\partial S} & =\int_{B} w \mathrm{~d} v=\frac{g_{2}}{2 \pi i}\left[\left(\Lambda_{0}-c\right)^{2}-\frac{\mu}{2}-\frac{\mu}{2} \log \left(\frac{4 \Lambda_{0}^{2}}{\mu}\right)\right]+\mathcal{O}\left(\log \Lambda_{0} / \Lambda_{0}\right) \\
& =W\left(\Lambda_{0}\right)+S-S \log \left(\frac{S}{-2 g_{2} \Lambda_{0}^{2}}\right)
\end{aligned}
$$


The superpotential (49) is equal to

$$
W=N S-N S \log \left(\frac{S}{-2 g_{2} \Lambda_{0}^{2}}\right)-2 \pi i \tau S
$$

where we dropped a constant term proportional to $W\left(\Lambda_{0}\right)$. By comparing this to the on-shell answer, we see that with our conventions the relation between $\tau$ and $\Lambda_{0}$ should be

$$
2 \pi i \tau=N \log \left(\frac{-2 g_{2} \Lambda_{0}^{2}}{\Lambda_{N=1}^{3}}\right)=N \log \left(\frac{-2 \Lambda_{0}^{2}}{\Lambda_{N=2}^{2}}\right),
$$

where we used the scale matching $\Lambda_{N=1}^{3}=g_{2} \Lambda_{N=2}^{2}$ between the high-energy $\mathcal{N}=2$ scale $\Lambda_{N=2}$ and the low-energy $\mathcal{N}=1$ scale $\Lambda_{N=1}$. This is exactly the reason why we chose the normalization of $\tau$ as in (59). Using (79), the superpotential reduces to the standard Veneziano-Yankielowicz result

$$
W=R N S-R S \log \left(\frac{S^{N}}{\Lambda_{N=1}^{3 N}}\right),
$$

and one recovers all familiar results for the $\mathcal{N}=1$ theory.

Combining (71), (72), (76) and (79), we obtain the following equation for $b$

$$
b \log \left(\frac{4 \Lambda_{0}^{2} \bar{\Lambda}_{0}^{2} g_{2}^{2}}{S \bar{S}}\right)=\log \left(\frac{S^{N}}{\Lambda_{N=1}^{3 N}}\right) .
$$

This result tell us that on-shell $b=0$, since the right hand side of this equation is then identically zero. However, off-shell $b$ depends non-trivially on $S, \bar{S}$, but also on $\Lambda_{0}$. As we take the cutoff to infinity, $b$ becomes smaller and smaller, but this is an example where computing the superpotential and taking the cutoff to infinity are not two commuting operations. One should first regulate the problem, then compute everything, and finally take the cutoff to infinity. There is therefore no simple non-holomorphic surface that gives the right answer without the need to introduce a cutoff. Finite non-holomorphic surfaces describe situations where the glueball superfields have expectation values of the order $|S| \sim\left|\Lambda_{0}\right|^{\eta}$ for some finite value of $\eta$.

The case where the superpotential is arbitrary but the Riemann surface still has only one cut can also be treated along similar lines, see e.g. [2] for more details about this situation.

In appendix B we illustrate the case of two cuts and comment on the general case.

\subsection{Alternative variational problem}

So far we have looked at the variational problem where deformations of the brane leave the asymptotics of the brane fixed. That is, we considered normalizable deformations, which are the ones of direct physical interest ${ }^{4}$. In principle, however, one can consider holomorphic but non-normalizable deformations and see what they correspond to in the gauge theory.

This is now straightforward. In this case $t$ is holomorphic, and $w$ contains non-normalizable deformations which correspond to changing the coefficients of the tree level superpotential. The conditions (20) are then reformulated as follows:

$$
\begin{aligned}
\hat{P}_{N}^{2}-\Lambda^{2 N} & =\hat{S}_{N-n}^{2}\left(\hat{G}_{n}+\hat{f}_{n-1}\right) \\
\hat{W}_{m}^{\prime 2}+\hat{\tilde{f}}_{m-1} & =\hat{H}_{m-n}^{2}\left(\hat{G}_{n}^{2}+\hat{f}_{n-1}\right) .
\end{aligned}
$$

\footnotetext{
${ }^{4}$ As remarked, because the Riemann surface was non-compact we actually had to include a non-holomorphic deformation with a logarithmic divergence.
} 
where again the hat denotes polynomials whose coefficients are to be determined from the equations. Again, we need at least $n$ free undetermined coefficients to vary the $S_{i}$ 's. A simple counting of equations shows that this is possible.

The above describe the only allowed holomorphic deformations if we want to keep the polynomial behavior of $t$ and $w$ in $v$ at infinity unmodified (even though we change the coefficients of $w$ ), and without introducing additional singularities in $t$. In conclusion, varying the $S_{i}$ 's will now mean varying the coefficients of $W_{m}^{\prime}$.

This is of course consistent with the fact that the glueball superfields can be obtained from the effective superpotential, $\left\langle S_{i}\right\rangle \sim \frac{\partial W_{\text {eff }}}{\partial \tau_{i}}$, as the latter contains the coefficients of $W_{m}^{\prime}$. In view of the partial integration that we did on Witten's original expression for the superpotential, it would be interesting to re-analyze the superpotential from this alternative perspective. Notice also that the non-normalizable deformations obtained by changing the tree-level superpotential of the high-energy theory are reminiscent of the non-normalizable deformations in AdS that correspond to the sources that couple to operators in the CFT.

\section{The superpotential and the $(2,0)$ theory}

One would like to see if the superpotential can be obtained directly in the $(2,0)$ theory living on the worldvolume of the fivebrane, as was suggested in [7]. For M5-brane configurations with $\mathcal{N}=2$ supersymmetry, where only one of the scalars $t$ or $w$ is active, it is well known $[12,14]$ that the equations of motion for the gauge fields on the 5-brane correctly reproduce the Kähler potential of the $\mathcal{N}=2 \mathrm{SYM}$ theory. It is interesting to see in detail whether this relation generalizes to the $\mathcal{N}=1$ case. Of course, once we break supersymmetry we do not expect the Kähler potential to agree, but we should still be able to reproduce the F-terms and in particular the superpotential.

So in order to see if we can we get the above superpotential directly in the $(2,0)$ theory, let us briefly recall what our brane construction corresponds to in terms of the field content on the brane. The $(2,0)$ tensor multiplet consists of a $(1,0)$ tensor multiplet and a hypermultiplet. The bosonic field content of the $(1,0)$ tensor multiplet is an antisymmetric 2 -form potential $B_{M N}$ with self-dual three-form field strength, and a real scalar $\phi$. The hypermultiplet contains four real scalars which can be combined into two complex scalars $\phi^{i}, i=1,2$. Thus, in all there are five real scalars which describe the transverse coordinates to the brane, in our set-up $t, w$, and $X^{9}$ which we set to zero. Thus only two complex scalars are non-trivial, and these combine together with the fermions in a four-dimensional hypermultiplet, which we will denote by $\Phi^{i}$, $i=1,2$, following the notation used earlier for the scalars. However, now one should interpret these as two $\mathcal{N}=1$ superfields that make up an $\mathcal{N}=2$ hypermultiplet. By construction, all fields in the tensor multiplet are trivial and set to constant values so we will ignore them for the time being.

To see how to get the superpotential, we can follow the recipe in $[28,29]$ of rewriting the 6 -dimensional fields in terms of an infinite family of 4-dimensional $\mathcal{N}=1$ superfields labeled by a continuous parameter, which in this case will be the complex coordinate $v$ which parametrizes the dependence of the fields on the internal manifold $\Sigma$. Using the expressions in [28], the scalar 
part of the action reads ${ }^{5}$

$$
S\left[\phi^{i}\right]=\int \mathrm{d}^{4} x \mathrm{~d}^{2} v \mathrm{~d}^{2} \theta \Phi^{1} \bar{\partial} \Phi^{2} .
$$

There is a choice here which complex coordinate we associate with $\Phi^{1}$ and which one with $\Phi^{2}$. On a compact Riemann surface both choices are equivalent, but here we are dealing with a noncompact surface and the two choices are different. As we explained before, below (40), this is determined by the boundary conditions, and therefore we will associate $\Phi^{1}$ with $w$ and $\Phi^{2}$ with $\log t$. With this choice we now recognize that (83) is the same as our previous expressions (40) and (51) for the superpotential. Notice that in (83) we can replace $\bar{\partial} \log t \mathrm{~d} \bar{v}$ by $\mathrm{d} \log t$, since the holomorphic piece in $\mathrm{d} \log t$ does not contribute to the integral.

Clearly, it would be interesting to study this deconstruction in more detail and to see whether other terms in the effective action have an equally nice geometric interpretation.

\section{The resolvent and the gauge kinetic terms}

In this section we briefly comment on some other physical quantities, namely the gauge theory resolvent and its Kähler potential, and their relation to the fivebrane theory.

On-shell, the gauge theory resolvent was simply given by $\mathrm{d} t / t$, see equations (4)-(6). Offshell, this can no longer be true, since the gauge theory resolvent is holomorphic whereas $t$ becomes non-holomorphic. It is however easy to see from (5) that the A-periods of the gauge theory resolvent will off-shell still be $N_{i}$. Therefore, the gauge theory resolvent is given by the unique holomorphic one-form whose A-periods are $N_{i}$. The B-periods of the gauge theory resolvent will no-longer be integer, but that is not important since the gauge theory resolvent need not have a straightforward geometrical interpretation. To compute the gauge theory resolvent we can either construct directly a suitable one-form, or we can use

$$
\operatorname{Tr}_{\text {gaugetheory }}\left(\frac{\mathrm{d} v}{v-\Phi}\right)=\sum_{i} N_{i} \frac{\partial}{\partial S_{i}} w \mathrm{~d} v
$$

which follows from (5) and (6), or equivalently we can see this directly from (4). It would be interesting to understand the gauge theory resolvent more directly from the fivebrane point of view.

Let us next consider the Kähler potential of the low-energy effective theory on the five-branes, as considered in $[12,14]$. We will mostly follow the computation of [12]. The Kähler potential is not protected by supersymmetry and therefore we don't expect the answer that we find to be in agreement with the Kähler potential in the pure gauge theory. Nevertheless, it may share some qualitative features with the gauge theory answer and it is therefore worth exploring.

Since the off-shell fivebrane configuration is non-holomorphic, the induced metric on it will not be proportional to $\mathrm{d} v \mathrm{~d} \bar{v}$, but also have $\mathrm{d} v \mathrm{~d} v$ and $\mathrm{d} \bar{v} \mathrm{~d} \bar{v}$ components. In principle, it would be nicer to introduce different coordinates on the Riemann surface in terms of which the metric is in conformal gauge, but in practice it may be difficult to explicitly construct such coordinates.

\footnotetext{
${ }^{5}$ We are using a linearized version of the Dirac-Born-Infeld action here, keeping only the quadratic terms. See also the next section.
} 
To write the kinetic term for the glueball fields, we write the metric in the internal directions as

$$
G_{p q} \mathrm{~d} X^{p} \mathrm{~d} X^{q}=\mathrm{d} v \mathrm{~d} \bar{v}+\mathrm{d} w \mathrm{~d} \bar{w}+R^{2} \mathrm{~d} \log t \mathrm{~d} \log \bar{t} .
$$

We will also write $v^{\alpha}=(v, \bar{v})$, and for the Riemann surface take a family that varies over $\mathbb{R}^{4}$, i.e. one where the glueball fields are non-constant. In other words, we take as our Riemann surface $X^{p} \equiv X^{p}\left(v, \bar{v}, S^{i}\left(x^{\mu}\right), \bar{S}^{i}\left(x^{\mu}\right)\right)$.

We define

$$
\begin{aligned}
E_{\mu}^{p} & =\partial_{\mu} S^{i} \partial_{i} X^{p}+\partial_{\mu} S^{\bar{i}} \partial_{\bar{i}} X^{p} \\
g_{\alpha \beta} & =\partial_{\alpha} X^{p} G_{p q} \partial_{\beta} X^{q}
\end{aligned}
$$

and

$$
H_{p q}=G_{p r} \partial_{\alpha} X^{r} g^{\alpha \beta} \partial_{\beta} X^{s} G_{s q}
$$

The metric $g_{\alpha \beta}$ is the induced metric on the Riemann surface. The kinetic terms for $S^{i}, \bar{S}^{i}$, as obtained from the Born-Infeld action, now read

$$
S_{\mathrm{kin}}=\int d^{4} x d^{2} v \sqrt{-\operatorname{det} g_{\alpha \beta}} \eta^{\mu \nu} E_{\mu}^{p}\left(G_{p q}-H_{p q}\right) E_{\nu}^{q} .
$$

This is a rather complicated expression due to the fact that the fivebrane configurations are nonholomorphic. A puzzling feature is the appearance of kinetic terms of the form $\partial_{\mu} S^{p} \partial^{\mu} S^{q}$ that include two holomorphic fields $S$ (and similar terms with two antiholomorphic glueball fields). In an $\mathcal{N}=1$ theory, one would expect only terms of the form $\partial_{\mu} S^{p} \partial^{\mu} \bar{S}^{q}$. Here, the fivebrane configuration explicitly breaks $\mathcal{N}=1$ supersymmetry, and this is why terms like $\partial_{\mu} S^{p} \partial^{\mu} S^{q}$ are being generated. It is possible that this is an artifact of the fivebrane theory, or that due to some mysterious cancellation these terms disappear after integrating over the Riemann surface. In any case, we cannot derive a Kähler potential from (88). The only way to obtain a Kähler potential from (88) is to ignore all $\bar{v}, \bar{S}$ dependence in $\log t$. That leads to a Kähler potential

$$
K \sim \int \mathrm{d}^{2} v k \partial_{v} \partial_{\bar{v}} k
$$

where

$$
k=|v|^{2}+|w|^{2}+R^{2}|\log t|^{2}
$$

is the space-time Kähler potential, now viewed as a function of $v, \bar{v}, S, \bar{S}$. There may be some relation between the field theory Kähler potential and (89), but there is no a priori reason to believe such a relation exists.

\section{Conclusion}

In this paper we have argued that the off-shell deformations of the M5-brane that correspond to turning on non-trivial expectation values for the glueball fields are particular non-holomorphic ones. We have expressed these in terms of the periods of differentials made out of the embedding coordinates and used this to compute the superpotential of these theories using the definition 
of the superpotential given by Witten in [5]. This definition involves the integral over a threemanifold that bounds two different Riemann surfaces and can be viewed as computing a domain wall tension, i.e. the difference between the value of the superpotential at two different vacua. By partial integration, it can be written as an integral over a two-form on $\Sigma$; such a form was also used in e.g. [23, 22, 30]. This form has many interpretations. It can be viewed as the contribution of a $6 \mathrm{~d}$ hypermultiplet to the superpotential as we discussed above, equivalently one can obtain it from a dimensional reduction of the type IIB superpotential $\int H \wedge \Omega$. All these give the same answer for the superpotential. We also saw that after carefully taking the boundary conditions into account, we could recover both the DV-version of the superpotential, as well as a more direct description in terms of the gauge theory resolvent.

Altogether, we see that the single M5-brane picture provides a setup where everything has become geometrical, and in particular all quantum information has become geometrical. The computations bear a close resemblance to the computations in IIB string theory with fluxes where one employs the superpotential $\int H \wedge \Omega$. In fact, the IIB string theory and the M5-brane configuration are T-dual to each other; under this T-duality, non-trivial IIB geometry and flux are replaced by a trivial background geometry with a non-trivial brane configuration.

The approach in [1] is somewhat intermediate, in that part of the quantum dynamics is encoded in the matrix model that arises. It is not quite clear in what sense this approach, which uses topological string theory, is dual to or can be embedded directly in the M5-brane setup. The action of the matrix model itself is similar to the IIA brane configuration, in that it only has classical information, and doing the matrix integral is analogous to the lift of the IIA configuration to M-theory. The precise relation between the M5-brane physics and the topological string theory is clearly something worth pursuing further.

There are many directions in which the results of this paper can be generalized. It should be relatively straightforward to generalize these results to other gauge theories for which a IIA brane description is known, such as theories with matter, quiver gauge theories and theories with $S O(N) / S P(N)$ gauge groups. It would certainly also be worthwhile to see to what extent non-holomorphic deformations of other wrapped brane configurations are physically relevant. We also believe that this work should have some relation to and implications for more general topological string backgrounds such as the the ones considered in [31,32], as well as for the approach to $\mathcal{N}=2$ theories advocated in [33]. We hope to return to some of these issues in the near future.

\section{Acknowledgments}

We would like to thank Robbert Dijkgraaf and Annamaria Sinkovics for collaboration and useful discussions during the early stages of this work. We would also like to thank Mina Aganagic, Tim Hollowood and Cumrun Vafa for valuable discussions and suggestions. SdH thanks the Simons Workshop on Mathematics and Physics, where part of this work was carried out, and the Institute for Theoretical Physics of the University of Amsterdam for hospitality.

This material is based upon work supported by the National Science Foundation under Grant No. PHY-0099590. Any opinions, findings, and conclusions or recommendations expressed in this material are those of the authors and do not necessarily reflect the views of the National 


\section{A Definition of the period integrals}

Integrals along paths going from one branch of the Riemann surface to the other are a priori not well defined, as one in addition needs to specify the path. Here we briefly give our definition of these integrals by a definition of the A- and B-periods that we use in practical computations.

A basis of compact A-cycles is provided by the cycles $A_{i}, i=1, \ldots, n-1$, encircling a single cut in the counterclockwise direction without intersecting. In our applications, however, we also need to take into account the points at infinity, $P$ and $\tilde{P}$, and so we will have to supplement this with integrals around $P$. Notice that in our applications the sum over all A-periods, including $A_{n}$, can be shown to be equal to the contour around $P$ by a contour deformation. This (infinite) contour is defined by $\Lambda_{0} \rightarrow e^{2 \pi i} \Lambda_{0}$, where $\Lambda_{0}$ is a regulator that we take to infinity at the end.

Concretely, we compute A-periods by taking the contours counterclockwise and defining the integrals with the appropriate signs, depending on which branch we are on:

$$
\oint_{A_{i}}=\int_{\tilde{c}_{i}^{-}}^{\tilde{c}_{i}^{+}}+\int_{c_{i}^{+}}^{c_{i}^{-}},
$$

$i=1, \ldots, n$. In most applications we encounter, these integrals add up to give a factor of 2 .

We can choose a set of compact B-cycles $\left\{B_{1}, \ldots, B_{n-1}\right\}$ defined as the cycles going from one cut to the other in the clockwise direction:

$$
\oint_{B_{i}}=\int_{c_{i}^{+}}^{c_{i+1}^{-}}+\int_{\tilde{c}_{i+1}^{-}}^{\tilde{c}_{i}^{+}},
$$

$i=1, \ldots, n-1$. Also here we need to supplement this with a non-compact period, which we take as going from $\tilde{P}$ to $P$ passing through $c_{n}^{+}$and appropriately regularized:

$$
\int_{B_{n}}=\int_{\tilde{\Lambda}_{0}}^{\tilde{c}_{n}^{+}}+\int_{c_{n}^{+}}^{\Lambda_{0}} .
$$

In the case where $n=1$, this will be the only period and we will call it simply $B$.

\section{B The case with more than one cut}

In this appendix we illustrate the method outlined in section 4 for surfaces with more cuts. We look at the case corresponding to the symmetry breaking pattern $U(N) \rightarrow U\left(N_{1}\right) \times U\left(N_{2}\right)$ in the field theory, or $n=2$. So we write

$$
y^{2}(v)=\left(v-c_{1}^{-}\right)\left(v-c_{1}^{+}\right)\left(v-c_{2}^{-}\right)\left(v-c_{2}^{+}\right)
$$

In this case we will need the following two basic integrals in order to solve for $T$ :

$$
\begin{aligned}
& \int \frac{\mathrm{d} v}{y(v)} \\
& \int \frac{v \mathrm{~d} v}{y(v)}
\end{aligned}
$$


These integrals are given in terms of elliptic integrals of the first and third kind ${ }^{6}$.

For simplicity we will work in the classical limit, where the distance between the two cuts is much larger than their length, and we take $c_{i}^{ \pm}=c_{i} \pm \mu_{i}$, where $\mu$ is a small parameter related to the coefficients $f_{i}$. Our approximation will be an expansion in $\frac{\mu_{i}}{c_{1}-c_{2}}$. In the field theory, since roughly $\mu \sim \Lambda_{N=2}^{2 N}$, this corresponds to the classical limit of small mass scale $\Lambda_{N=2}$.

We can write an integral over the first cut as:

$$
\oint_{A_{1}} \frac{\mathrm{d} v}{y(v)}=\frac{2}{c_{1}-c_{2}} \int_{-\sqrt{\mu_{1}}}^{\sqrt{\mu_{1}}} \frac{\mathrm{d} x}{\sqrt{x^{2}-\mu_{1}}}+\mathcal{O}\left(\frac{\mu_{1}}{c_{1}-c_{2}}\right)=-\frac{2 \pi i}{c_{1}-c_{2}}+\mathcal{O}\left(\frac{\mu_{1}}{c_{1}-c_{2}}\right)
$$

where we took the period in the counterclockwise direction. Obviously, there is a similar contribution around $A_{2}$. We also need the following integral:

$$
\oint_{A_{i}} \frac{v \mathrm{~d} v}{y(v)}=c_{i} \oint_{A_{i}} \frac{\mathrm{d} v}{y(v)}+\mathcal{O}\left(\frac{\mu_{i}}{c_{i}-c_{j}}\right)
$$

Writing

$$
T=\left(a_{0}+a_{1} v\right) \frac{\mathrm{d} v}{y(v)}+\left(b_{0}+b_{1} \bar{v}\right) \frac{\mathrm{d} \bar{v}}{\bar{y}(\bar{v})}
$$

and taking into account all contributions we get

$$
N_{i}=-\frac{a_{0}+a_{1} c_{i}}{c_{i}-c_{j}}+\frac{b_{0}+b_{1} \bar{c}_{i}}{\bar{c}_{i}-\bar{c}_{j}}+\mathcal{O}\left(\frac{\mu}{c_{i}-c_{j}}\right)
$$

where obviously $j \neq i$.

The $\Lambda_{0}$-dependence of the non-compact period $B_{2}$ can be computed as before in powers of $\log \Lambda_{0} / \Lambda_{0}$. We get:

$$
\int_{\tilde{\Lambda}_{0}}^{\Lambda_{0}} T=2 a_{1} \log \Lambda_{0}+2 b_{1} \log \bar{\Lambda}_{0}+\mathcal{O}\left(\log \Lambda_{0} / \Lambda_{0}\right)=2 \pi i \tau
$$

This, together with (99) and the integral over $B_{1}$, which is again given by an elliptic integral, determines $a_{0}, a_{1}, b_{0}$ and $b_{1}$ uniquely.

Let us make some comments about the general case, $U(N) \rightarrow \prod_{i=1}^{n} U\left(N_{i}\right)$. As the genus of the curve goes up, the integrals that one needs to solve get more and more involved. It is however not hard to see how the general case works. One needs to impose:

$$
\begin{aligned}
& \oint_{A_{i}} \frac{a_{n-1}(v)}{y(v)} \mathrm{d} v+\oint_{A_{i}} \frac{b_{n-1}(\bar{v})}{\bar{y}(\bar{v})} \mathrm{d} \bar{v}=2 \pi i N_{i} \\
& \oint_{B_{i}} \frac{a_{n-1}(v)}{y(v)} \mathrm{d} v+\oint_{B_{i}} \frac{b_{n-1}(\bar{v})}{\bar{y}(\bar{v})} \mathrm{d} \bar{v}=2 \pi i \tau_{i}
\end{aligned}
$$

both for the normalizable periods and for the non-normalizable one. The integrals over the normalizable periods are integers, as follows from the fact that $T$ is on-shell a meromorphic

\footnotetext{
${ }^{6}$ As an illustration, it is useful to first consider the case where the minima $c_{i}$ are such that we can use the following parametrization: $y=\sqrt{\left(t^{2}-\mu_{1}\right)\left(t^{2}-\mu_{2}\right)}$, which is consistent with the fact that $f_{n-1}(v)$ has two coefficients in this case. Using rescalings we can rewrite the integrals over the periods in terms of $\int_{0}^{1} \frac{\mathrm{d} v}{\sqrt{\left(1-v^{2}\right)\left(1-k^{2} v^{2}\right)}}=K(k)$ and $\int_{1}^{1 / k} \frac{\mathrm{d} v}{\sqrt{\left(1-v^{2}\right)\left(1-k^{2} v^{2}\right)}}=i K^{\prime}(k)$, where $K$ and $K^{\prime}$ are the usual complete elliptic integrals of the first kind [34]. The second integral in (95) can be explicitly computed in terms of a simple logarithm.
} 
differential that is written locally as $\mathrm{d} \log t$ where $t$ is the embedding coordinate. And even though $T(v, \bar{v})$ takes on a similar form by the relations (66), it is crucial that $\alpha(v)$ and $\beta(\bar{v})$ are not rational functions. Indeed, as we have seen explicitly, the exponents $a$ and $b$ are not integers, but they can take any values as functions of $N, \Lambda_{0}, \mu$, and $c$.

It is now useful to see whether the conditions (101) have solutions for arbitrary values of $n$. One can easily see that this is the case. They form $2 n$ conditions, and we have $2 n$ coefficients, $\left\{a_{1}, \ldots, a_{n}\right\}$ and $\left\{b_{1}, \ldots, b_{n}\right\}$, to adjust, so the solution, if there is one, is unique. It is thus crucial that we also include one off-shell log-normalizable deformation $b_{n-1}$.

On-shell, the $B$-periods are fixed once the complex structure $f_{i}, i=0, \ldots, n-1$, is fixed. In that case we can set all $b_{n-1}(\bar{v})=0$, and we are left with the $n$ parameters of $a_{n-1}(v)$, as we showed in section 4 .

The general case can be done without much more effort. If one wants to stay completely general, the expressions become complicated elliptic integrals. However, one can easily approximate these integrals in the semi-classical limit we have been considering before. We will skip details and just quote the result for the glueball expectation values:

$$
S_{i}=H_{m-n}\left(c_{i}\right) \frac{f_{n-1}\left(c_{i}\right)}{2 G_{n}^{\prime}\left(c_{i}\right)}
$$

Using the monodromy argument in [2] one also finds

$$
\tilde{f}_{m-1}=2 \sum_{i=1}^{n} S_{i}=\sum_{i=1}^{n} H_{m-n}\left(c_{i}\right) \frac{f_{n-1}\left(c_{i}\right)}{G_{n}^{\prime}\left(c_{i}\right)} .
$$

One can compute the A-periods of $T$ and the leading $\Lambda_{0}$-dependence of the $B$-periods of $T$ and $w \mathrm{~d} v$ similarly.

\section{References}

[1] R. Dijkgraaf and C. Vafa, "Matrix models, topological strings, and supersymmetric gauge theories," Nucl. Phys. B 644, 3 (2002) [arXiv:hep-th/0206255]; "On geometry and matrix models," Nucl. Phys. B 644, 21 (2002) [arXiv:hep-th/0207106]; "A perturbative window into non-perturbative physics," arXiv:hep-th/0208048.

[2] F. Cachazo, K. A. Intriligator and C. Vafa, "A large N duality via a geometric transition," Nucl. Phys. B 603, 3 (2001) [arXiv:hep-th/0103067].

[3] F. Cachazo, N. Seiberg and E. Witten, "Phases of $N=1$ supersymmetric gauge theories and matrices," JHEP 0302, 042 (2003) [arXiv:hep-th/0301006].

[4] F. Cachazo, N. Seiberg and E. Witten, "Chiral Rings and Phases of Supersymmetric Gauge Theories," JHEP 0304, 018 (2003) [arXiv:hep-th/0303207].

[5] E. Witten, "Solutions of four-dimensional field theories via M-theory," Nucl. Phys. B 500, 3 (1997) [arXiv:hep-th/9703166].

[6] K. Hori, H. Ooguri and Y. Oz, "Strong coupling dynamics of four-dimensional N = 1 gauge theories from M Adv. Theor. Math. Phys. 1, 1 (1998) [arXiv:hep-th/9706082]. 
[7] E. Witten, "Branes and the dynamics of QCD," Nucl. Phys. B 507, 658 (1997) [arXiv:hepth/9706109].

[8] A. Giveon and D. Kutasov, "Brane dynamics and gauge theory," Rev. Mod. Phys. 71, 983 (1999) [arXiv:hep-th/9802067].

[9] J. de Boer and Y. Oz, "Monopole condensation and confining phase of $\mathrm{N}=1$ gauge theories via Nucl. Phys. B 511, 155 (1998) [arXiv:hep-th/9708044].

[10] C. Vafa, "Superstrings and topological strings at large N," J. Math. Phys. 42, 2798 (2001) [arXiv:hep-th/0008142]; R. Gopakumar and C. Vafa, "On the Gauge Theory/Geometry Correspondence", Adv. Theor. Math. Phys. 3(1999)1415-1443, [arXiv: hep-th/9811131]

[11] K. Dasgupta, K. Oh and R. Tatar, Nucl. Phys. B 610, 331 (2001) [arXiv:hep-th/0105066].

[12] J. de Boer, K. Hori, H. Ooguri and Y. Oz, "Kaehler potential and higher derivative terms from M theory five-brane," Nucl. Phys. B 518, 173 (1998) [arXiv:hep-th/9711143].

[13] S. k. Nam, K. Oh and S. J. Sin, "Superpotentials of $N=1$ supersymmetric gauge theories from M-theory," Phys. Lett. B 416, 319 (1998) [arXiv:hep-th/9707247].

[14] P. S. Howe, N. D. Lambert and P. C. West, "Classical M-fivebrane dynamics and quantum N = 2 Yang-Mills," Phys. Lett. B 418, 85 (1998) [arXiv:hep-th/9710034].

[15] F. Cachazo, M. R. Douglas, N. Seiberg and E. Witten, JHEP 0212, 071 (2002) [arXiv:hepth/0211170].

[16] S. Elitzur, A. Giveon and D. Kutasov, "Branes and N = 1 duality in string theory," Phys. Lett. B 400, 269 (1997) [arXiv:hep-th/9702014].

[17] S. Elitzur, A. Giveon, D. Kutasov, E. Rabinovici and A. Schwimmer, "Brane dynamics and N = 1 supersymmetric gauge theory," Nucl. Phys. B 505, 202 (1997) [arXiv:hepth/9704104].

[18] I. A. Bandos, K. Lechner, A. Nurmagambetov, P. Pasti, D. P. Sorokin and M. Tonin, "Covariant action for the super-five-brane of M-theory," Phys. Rev. Lett. 78, 4332 (1997) [arXiv:hep-th/9701149].

[19] M. Aganagic, J. Park, C. Popescu and J. H. Schwarz, "World-volume action of the M-theory five-brane," Nucl. Phys. B 496, 191 (1997) [arXiv:hep-th/9701166].

[20] P. Arvidsson, E. Flink and M. Henningson, "Free tensor multiplets and strings in spontaneously broken six-dimensional JHEP 0306, 039 (2003) [arXiv:hep-th/0306145].

[21] M. Bershadsky, C. Vafa and V. Sadov, "D-Branes and Topological Field Theories," Nucl. Phys. B 463, 420 (1996) [arXiv:hep-th/9511222].

[22] S. Kachru, S. Katz, A. E. Lawrence and J. McGreevy, Phys. Rev. D 62, 026001 (2000) [arXiv:hep-th/9912151]. 
[23] M. Aganagic and C. Vafa, "Mirror symmetry, D-branes and counting holomorphic discs," [arXiv:hep-th/0012041].

[24] F. Cachazo and C. Vafa, "N=1 and N = 2 geometry from fluxes," [arXiv:hep-th/0206017].

[25] H. M. Farkras and I. Kra, "Riemann Surfaces", Springer-Verlag 1991.

[26] T. J. Hollowood, "Critical points of glueball superpotentials and equilibria of integrable systems," JHEP 0310, 051 (2003) [arXiv:hep-th/0305023].

[27] R. Boels, J. de Boer, R. Duivenvoorden and J. Wijnhout, "Factorization of Seiberg-Witten curves and compactification to three dimensions," [arXiv:hep-th/0305189].

[28] N. Arkani-Hamed, T. Gregoire and J. Wacker, "Higher dimensional supersymmetry in 4D superspace," JHEP 0203, 055 (2002) [arXiv:hep-th/0101233].

[29] R. Dijkgraaf and C. Vafa, "N = 1 supersymmetry, deconstruction, and bosonic gauge theories," [arXiv:hep-th/0302011].

[30] W. Lerche, P. Mayr and N. Warner, "Holomorphic N = 1 special geometry of open-closed type II strings," [arXiv:hep-th/0207259]; W. Lerche, P. Mayr and N. Warner, "N = 1 special geometry, mixed Hodge variations and toric geometry," [arXiv:hep-th/0208039].

[31] M. Aganagic, A. Klemm, M. Marino and C. Vafa, "The topological vertex," [arXiv:hepth/0305132].

[32] M. Aganagic, R. Dijkgraaf, A. Klemm, M. Marino and C. Vafa, "Topological strings and integrable hierarchies," [arXiv:hep-th/0312085].

[33] N. Nekrasov and A. Okounkov, "Seiberg-Witten theory and random partitions," [arXiv:hepth/0306238].

[34] D. F. Lawden, "Elliptic Functions and Applications", Springer-Verlag, 1980. 